\title{
A review of input data and modelling assumptions in longitudinal strength models for unidirectional fibre-reinforced composites
}

\author{
Yentl Swolfs*, Ignaas Verpoest, Larissa Gorbatikh \\ Department of Materials Engineering, KU Leuven \\ Kasteelpark Arenberg 44 bus 2450 \\ 3001 Leuven - Belgium \\ *Corresponding author: yentl.swolfs@mtm.kuleuven.be
}

Fibre-reinforced composites are rapidly increasing their market share in structural applications. Nevertheless, this increase would be much stronger if reliable failure predictions were available. These predictions are not only insufficiently reliable for complex loading of multidirectional composites, but even for longitudinal tensile failure of unidirectional (UD) composites. Since composite failure usually coincides with longitudinal failure of a $0^{\circ}$ ply, the reliability often hinges on longitudinal failure predictions of UD composites. Despite great progress in the state-of-the-art models, significant obstacles remain in collecting the necessary input data and understanding the influence of the modelling assumptions. This review therefore surveys the mechanics, chemistry and physics involved in tensile failure of UD composites and highlights potential areas for improvement. Specific proposals are made to advance the state-of-the-art strength models, which could catalyse the use of composites in structural applications.

Keywords: Tensile failure; Modelling; Stress concentrations; Fibre strength, Weibull distribution; Fibre breaks

\section{Introduction}

Predictive models for mechanical properties are crucial for designing composite applications in an optimal manner. Even though some stiffness variations are unavoidable in real parts [1], predictions of composite stiffness are often used with confidence in industrial applications. Predictions of strength and damage development, however, remain highly challenging. The World Wide Failure Exercise I, II and III therefore attempted to predict failure of multidirectional composites under complex loading conditions [2-4]. A large spread on the modelling predictions was found, indicating the reason for the lack of confidence of industry in models. The large spread was attributed to the complex internal structure of fibrereinforced composites and complex interactions between fibre and matrix.

The longitudinal tensile failure and damage development in $0^{\circ}$ unidirectional (UD) composites is better understood. It is also crucial in the failure of multidirectional composites, as final failure usually coincides with failure of the $0^{\circ}$ plies. A key concept in longitudinal tensile failure of UD composites is that the fibres in a bundle do not all possess exactly the same strength. Instead, the strength of these fibres typically follows a Weibull distribution [5]. This fibre strength variation is crucial in the longitudinal tensile failure development of UD composites. Upon increasing the applied strain, the weakest fibres fail first. Each broken fibre locally stops carrying load and sheds that load to the nearby fibres. The matrix surrounding the fibre break is loaded in shear and transfers stress back onto the broken fibre [6-10]. Therefore, the nearby fibres will locally carry stress concentrations, but the magnitude decreases with increasing distance from the fibre break [11-16]. 
A vital consequence of these stress concentrations is that they increase the failure probability of the nearby fibres. Eventually, this increased failure probability will lead to the development of fibre break clusters [17-20], which further intensify the stress concentrations. One of these clusters will reach a certain critical size, after which this cluster propagate unstably. This unstable propagation will rapidly cover the entire cross-section of the composite and hence cause final composite failure. Apart from fibre strength, the stress redistribution around fibre breaks is also a crucial parameter in the failure development of UD composites. For a given fibre type, this redistribution is governed by properties of the matrix and the fibre-matrix interface. The magnitude of the stress concentrations and the length over which they are significant is crucial in determining failure of a UD composite. Correctly capturing the fibre strength statistics and stress redistribution around a broken fibre is therefore crucial to the success of strength models for UD composites. If this is combined with the appropriate input data, then a good correlation with experiments should be achievable. There are however significant experimental and theoretical difficulties in obtaining reliable input data for both aspects. This review aims to provide guidelines on how these input parameters should be measured and in which cases they are important. It will also propose strategies to overcome these difficulties in the future.

Even in the ideal scenario that the fibre, matrix and interfacial properties are measured accurately, deviations from experimental measurements may still occur. Every state-of-the-art strength model inevitably requires a set of assumptions that limit its accuracy. The section "3. Modelling assumptions" hence provides an overview of the most important and common assumptions in strength models for UD composites. Guidelines are provided on the importance of the various assumptions whenever the literature offers sufficient information to do so.

The focus of this review is on the input data and the modelling assumptions. The goal is to describe both aspects in a generic way that is largely independent of the chosen modelling approach. Describing the models themselves is therefore outside the scope of this review, as doing this in a comprehensive manner would be nearly impossible.

Depending on the nature of the model, many different outputs can be obtained. The literature has focused mainly on the tensile strength of the composite. Recently however, the availability of synchrotron radiation computed tomography has sparked the interest in tracking individual fibre breaks and clusters of fibre breaks [17-25]. Whenever this review mentions the word predictions, it refers either to tensile strength predictions or to a combination of tensile strength and fibre break predictions. Specific references to fibre break predictions will always be explicitly indicated.

The majority of the cited studies deal with carbon fibres in a thermoset matrix. This was not a deliberate choice, but occurred nonetheless for two reasons. Firstly, there is a large body of knowledge on carbon fibres and its composites. Secondly, the higher performance of thermoset carbon fibre composites has sparked a larger interest in predictive models than for thermoset composites with other fibres. Metal and ceramic matrix composites can also reach high performance levels, but they are not used in tensile loading as often as thermoset carbon fibre composites. Consequently, the majority of the literature on input data measurements and models focused on carbon fibre and its composites. Nevertheless, there are important aspects to be learned from other fibres as well as from other research fields. This review therefore also looks at studies on other fibre types and from other fields. This is especially true for 
section "2.1. Fibre strength", as there is a great body of knowledge on the strength of ceramic fibres such as SiC fibres.

\section{Input data}

Predictions of any model depend strongly on the input data. This is even more so in strength models for UD composites, which are often dominated by the fibre strength parameters. The matrix and interfacial properties also play an important role, as they will determine the magnitude and extent of the stress concentrations. Many different types of properties can be plugged into strength models, making it crucial to understand the importance of each of them. This section reviews the most recent insights into the two most crucial input data: fibre strength and the matrix/interfacial properties.

\subsection{Fibre strength}

As mentioned by Timoshenko [26], Leonardo da Vinci was the very first to study the strength of materials as a function of their size. Da Vinci's ground-breaking experiments revealed that longer iron wires were weaker than shorter ones. These experiments were described at the end of the $15^{\text {th }}$ century, long before the Griffith established the fracture mechanics of this problem. Griffith suggested that the size dependency of strength could be explained based on the presence of microscopic flaws [27].

The strength of a single fibre cannot be captured in one single average value. The most commonly used reinforcement fibres, such as carbon and glass, are brittle and hence exhibit weakest-link characteristics. This means they break as soon as the weakest link is overloaded. The failure probability is thus linked to the presence of weak links. One obvious conclusion is that a shorter fibre will have a lower probability of having a weak link or flaw and is hence more likely to have a higher strength. This reasoning also explains Da Vinci's experiments.

The statistical function that is generally believed to describe this weakest-link behaviour is the Weibull distribution. This is not always the case, as there are additional requirements apart from weakest-link behaviour to obtain such distribution [28,29]. Todinov [29] for example, proved that its mathematical formulation is only correct if the probability that a flaw becomes critical is a power law of the applied stress. The rest of this review will assume this is indeed the case.

\subsubsection{Standard Weibull distributions}

The standard Weibull probability distribution function $P$ for fibre strength is:

$$
P\left(\sigma_{f}\right)=1-\exp \left(-\left(\frac{L}{L_{0}}\right)\left(\frac{\sigma_{f}}{\sigma_{0}}\right)^{m}\right),
$$

with $L$ being the characteristic gauge length, $L_{0}$ the reference gauge length, $\sigma_{f}$ the fibre strength, $\sigma_{0}$ the scale parameter and $m$ the shape parameter or Weibull modulus [5]. The parameters $\sigma_{0}$ and $L$ are typically calculated from single fibre tests at gauge lengths $L_{0}$ between 10 and $50 \mathrm{~mm}$. The overwhelming majority of strength models uses this standard Weibull distribution. The scale parameter $\sigma_{0}$ is the strength at which $63.2 \%$ of the fibres will fail (see Figure 1). The Weibull modulus $m$ determines the scatter around the average value. 
A large Weibull modulus indicates a narrow distribution, which corresponds to little scatter in the fibre strength (see Figure 1).

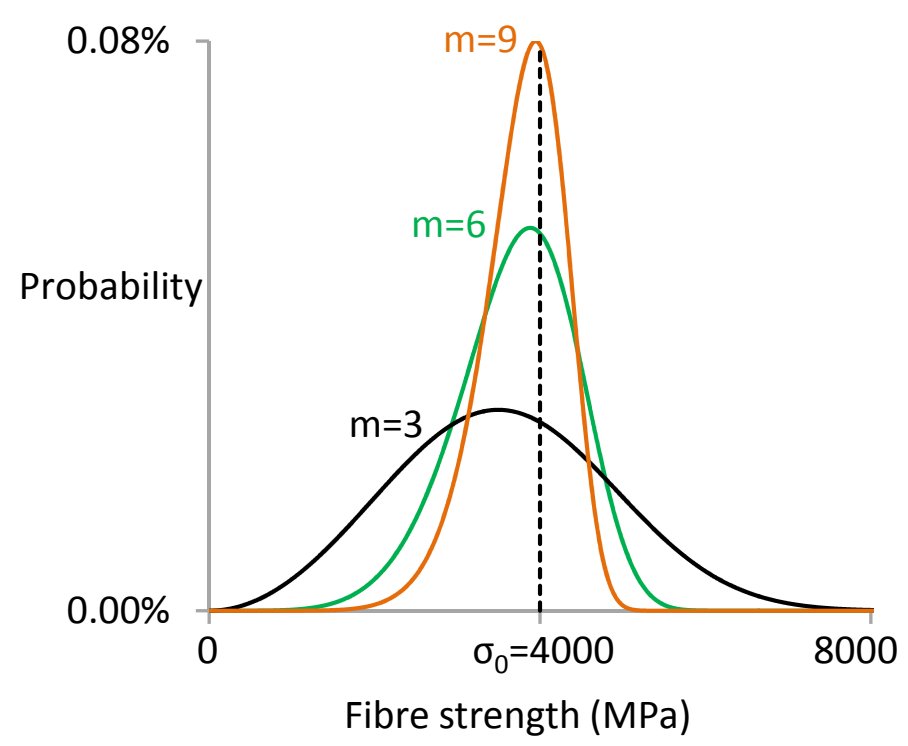

Figure 1. Example of a Weibull distribution with $\sigma_{0}=4000 \mathrm{MPa}$, showing how the scatter in fibre strength increases when the Weibull modulus decreases.

In the initial distribution proposed by Weibull himself [5], a lower limit $\sigma_{u}$ for the fibre strength was included:

$$
P\left(\sigma_{f}\right)=1-\exp \left(-\left(\frac{L}{L_{0}}\right)\left(\frac{\sigma_{f}-\sigma_{u}}{\sigma_{0}}\right)^{m}\right),
$$

This lower limit basically means that the strength of each fibre is at least equal to $\sigma_{u}$. It is extremely difficult to prove this point experimentally, as every test requires a minimum strength level to perform the test [30]. This distribution has been used by some authors [30,31], although most assume $\sigma_{u}$ equals zero. Its effect on the tensile strength predictions is unknown in the literature, as it is very difficult to reliably measure $\sigma_{u}$. The remainder of this review will therefore ignore this additional term.

Most strength models require the Weibull distribution at gauge lengths $L$ that are significantly smaller than $10-50 \mathrm{~mm}$ [31]. The relevant lengths are often determined by the slip or ineffective length (see "2.2. Matrix and interfacial properties"), which is on the order of magnitude of a $100 \mu \mathrm{m}$ [32-35]. Many models however require multiple partitions within this slip length, which can bring down the relevant length to just a few $\mu \mathrm{m}$ [20,22,23,36-39]. For this purpose, the Weibull distribution should be extrapolated to short gauge lengths. To get a better feeling for this extrapolation, the term $L / L_{0}$ can be re-arranged underneath the exponent $m$ as follows:

$$
P\left(\sigma_{f}\right)=1-\exp \left(-\left(\frac{\sigma_{f}}{\sigma_{0} \cdot L_{0}^{1 / m} / L^{1 / m}}\right)^{m}\right)
$$

The extrapolation therefore essentially means that the characteristic strength $\sigma_{0}^{*}$ at a gauge length $L$ equals: 


$$
\sigma_{0}^{*}=\sigma_{0} \cdot\left(\frac{L_{0}}{L}\right)^{1 / m}
$$

Several authors have mentioned that this extrapolation breaks down at short gauge lengths, typically around a few mm [31,40-43]. This break-down was attributed to three causes: (1) fibre diameter variations [41,44-48], (2) variations of the Weibull distribution from fibre-tofibre within the same bundle [41,44,49,50], and (3) the presence of different strengthdetermining flaw populations [40,43,51-53]. Explaining how the first two causes lead to underestimations at short gauge lengths requires an extensive mathematical treatment, which outside the scope of this review paper. Interested readers can refer to the corresponding literature [41,47-49]. The third cause leads to multimodal Weibull distributions, which will be described in section "2.1.2. Modified Weibull distributions".

The breakdown of equation (4) at short gauge lengths is the main reason why modified versions of the Weibull distribution were developed. The background of these distributions will be described in detail in the next subsection.

\subsubsection{Modified Weibull distributions}

The unimodal Weibull distribution in equation (1) is the simplest way to capture weakest link behaviour. The unimodal distribution inherently assumes there is only one flaw population present. This may not necessarily be true, as experimental evidence of different flaw types exists in the literature [53-55]. The flaws should also be randomly distributed over the fibres. This may not be entirely the case due to variations in fibre production and handling. One can imagine that the outer fibres in a bundle may have been subjected to more friction as well as slightly different processing temperature than the inner fibres.

Many authors have suggested that multiple strength-determining flaw populations exist [40,43,51,52,56-61]. This leads to multimodal Weibull distributions, of which the bimodal is the most popular one. This leads to two terms inside the exponential function:

$$
P\left(\sigma_{f}\right)=1-\exp \left(-\left(\frac{L}{L_{0}}\right)\left(\frac{\sigma_{f}}{\sigma_{0,1}}\right)^{m_{1}}-\left(\frac{L}{L_{0}}\right)\left(\frac{\sigma_{f}}{\sigma_{0,2}}\right)^{m_{2}}\right),
$$

where $\sigma_{0,1}$ and $\sigma_{0,2}$ are the scale parameters, and $m_{1}$ and $m_{2}$ are the Weibull moduli for the first and second flaw population, respectively. Thomason indicated that this bimodal distribution may also be caused by an inherent assumption in the Weibull distribution [30]. The Weibull distribution in equation (1) assumes that there is no threshold stress below which the failure probability is zero. This is a common assumption in brittle materials. If however, such a threshold stress does exist, then it can be proven that a bimodal distribution automatically arises in measurements.

The second mode of the Weibull distribution will determine the strength at short gauge lengths. The Weibull modulus $m_{2}$ of this mode is larger than $m_{1}$, and hence has a narrower strength distribution. Harlow and Phoenix [62] claimed that a bimodal distribution essentially puts an upper limit to the fibre strength at short gauge lengths. Harlow and Phoenix reached this conclusion by using a Weibull modulus of 50 for $m_{2}$. Recent measurements for two dissimilar carbon fibres, however, have indicated much smaller values: 12 for T700S [51] and 13 for T800S [63]. With these smaller values, the rate at which the strength at short gauge lengths levels off is significantly slower. While the strength distribution still levels off, the claim of Harlow and Phoenix is perhaps too strong. 
Another type of distribution adds an exponent $\alpha$ to equation (2) to capture the gauge length dependence of the Weibull distribution $[41,42,64]$ :

$$
P\left(\sigma_{f}\right)=1-\exp \left(-\left(\frac{L}{L_{0}}\right)^{\alpha}\left(\frac{\sigma_{f}}{\sigma_{0}}\right)^{m}\right) .
$$

This distribution is often referred to as the power law-accelerated Weibull distribution. Equation (6) reduces to equation (1) if $\alpha$ equals 1 . Values for $\alpha$ of 0.6 [42], 0.7 [64] and 0.9 [41] have been reported. A crucial consequence of this type of distribution is that the strength distribution shifts to lower strengths if $L$ is smaller than $L_{0}$, and vice versa. Modelling results will hence depend on the chosen length $L$.

It should be noted that an $\alpha$-value below 1 may also be caused by some of the reasons that will be mentioned in section "2.1.3. Experimental and statistical issues with single fibre testing". At short gauge lengths, clamping effects are more pronounced and fibre alignment is more difficult, which could contribute to the underestimation of fibre strength at short gauge lengths. The statistical variation in the Weibull distribution would also require a large number of tests to be performed. Finally, it is unclear whether the power law-accelerated distribution holds true at short gauge lengths.

One of the initial problems with the power law-accelerated distribution was that it was mainly based on curve fitting rather than a solid theoretical background. Curtin [49] partially resolved this by proving that equation (6) may also arise due to processing and handling of the fibres. Curtin therefore proposed the "Weibull of Weibulls" distribution. This distribution assumes that the strength distribution along a fibre follows the standard Weibull distribution (see equation (1)). Based on this Weibull distribution, a characteristic strength at a certain length $L$ can be calculated for each fibre. Curtin then assumed that the characteristic strengths of each fibre are different and also follow a Weibull distribution. Such differences can be attributed to processing and handling of the fibres. With these assumptions, the distribution essentially reverts to the power law-accelerated Weibull distribution in equation (6), but with a more sound physical background. The disadvantage of the "Weibull of Weibulls" distribution is that it is challenging to implement in strength models. For the details of this distribution, the reader is referred to Curtin's work [49].

Other distributions have also been proposed, but they have been used in only a few cases. The reader can refer to Padgett et al. [65], Gurvich et al. [66], Phani [67], and Ibnabdeljalil and Phoenix [68] for some examples. There are several reasons why these more complicated distributions have not become widely used. Firstly, some of the distributions are mathematically complex and difficult to use in practice. Secondly, not all these distributions have a sound mechanical basis, and some are purely based on curve fitting. Thirdly, these distributions all require additional parameters that need to be fitted to the experimental data. This necessitates the need for more experiments to maintain the same level of confidence in the fitted parameters. Finally, some analytical models can only deal with a standard unimodal Weibull distribution.

\subsubsection{Experimental and statistical issues with single fibre testing}

Experimentally determining the Weibull distribution is a challenging task. The single fibre test is by far the most common technique (see Figure 2a), and is described in several standards, such as BS ISO 11566 and ASTM C1557. The test starts by extracting a single 
fibre from a bundle and gluing it onto a paper frame. This frame is then gripped in a tensile machine, after which both legs of the paper frame are cut and the test is started (see Figure 2a). By testing a sufficient number of fibres and recording the strength, the Weibull distribution can be fitted through the data. This is typically done through a maximum likelihood estimation, which is deemed to be more accurate than least squares regression $[30,69,70]$.

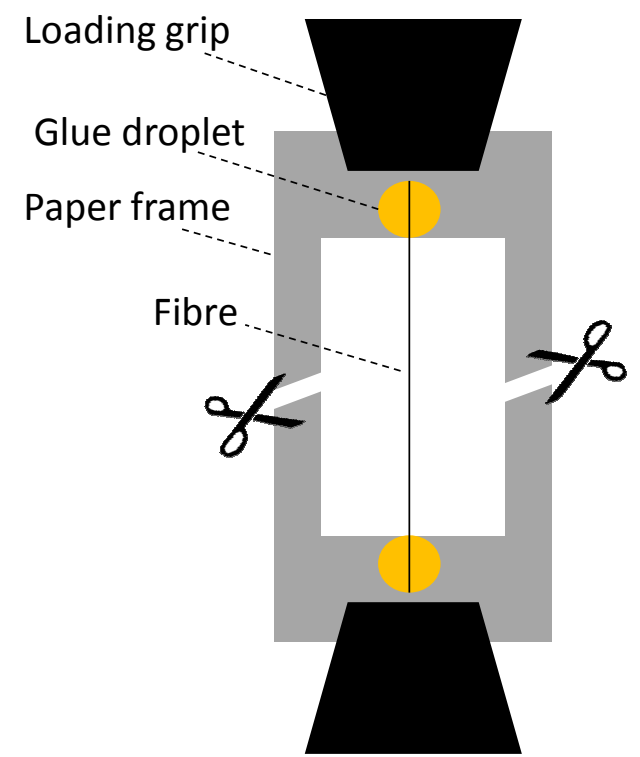

(a)

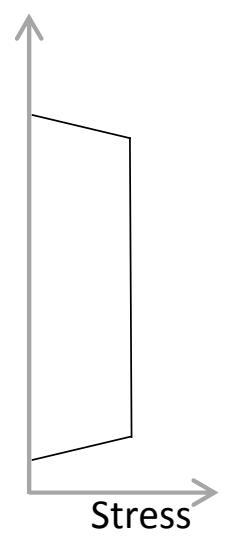

(b)

Figure 2. (a) Schematic illustration of a single fibre test with a paper frame that is cut prior to the test and two droplets of glue to transfer stress onto the fibre; (b) the stress profile this results in.

As will be shown later in this subsection, there are significant experimental and statistical issues in the determination of the Weibull parameters. As they are vital in strength models, this could also affect the reliability of strength models. Understanding the reasons behind the large variations in Weibull data is therefore crucial in advancing the state of the art. The next subsections describe various aspects that could contribute to these errors or that could help to resolve them in the future.

\section{Fibre preselection}

Most reinforcement fibres have a diameter on the order of $10 \mu \mathrm{m}$. Such small fibres lead to low breaking forces. A typical breaking force for a high strength carbon fibre is $0.15 \mathrm{~N}$. It is therefore not unlikely that some fibres break during the extraction from the bundle or during the cutting of the frame [30,46,71]. The elimination of the weakest fibres causes deviations from linearity in Weibull plots [47]. Such a deviation has been reported by many authors $[42,64,72,73]$ and an example is plotted in Figure 3. One can easily imagine that a distribution with more fitting parameters than the unimodal Weibull distribution would lead to a better fit. That fact of course does not imply that the basic assumptions, such as multiple flaw populations, are correct. 


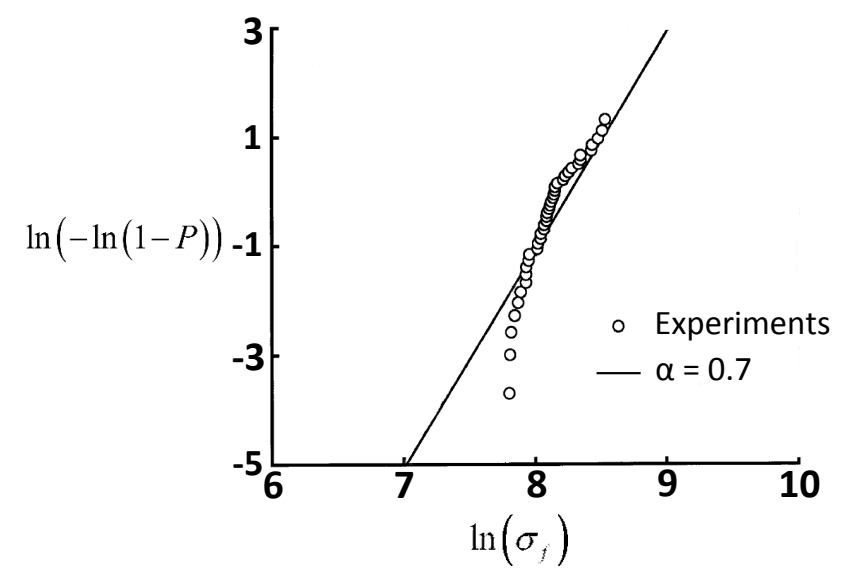

Figure 3. Weibull plot for $\mathbf{T 8 0 0 H}$ carbon fibre revealing the deviation from linearity at low strength. The data points indicate the experimental measurements from single fibre tests (Adapted from [64] with permission from Elsevier).

\section{Clamping effects in single fibre tests}

An ideal single fibre test would have a uniform tensile stress over the entire gauge length, and zero tensile stress outside of the gauge length. Clamping effects, which are defined here as deviations from this ideal scenario, always occur in single fibre testing (see Figure $2 b$ ) $[40,60,71,74]$. These effects are particularly pronounced at small gauge lengths for several reasons [74]. Firstly, fibre misalignment increases significantly at short gauge lengths. Misalignment introduces shear and bending near the adhesive, which increases the probability of failure in the clamps [71].

Secondly, the fibre stress needs to be built up through shear stresses between the glue and the fibre surface. The length of this build-up region $L_{\text {build-up }}$ can be estimated using perfect alignment and shear-lag assumptions, similar to the Kelly-Tyson model [75]. The equation for the stress build-up length becomes:

$$
L_{\text {build }-u p}=\frac{\sigma_{f, \max } \cdot \tau_{s}}{4 . D}
$$

with $\sigma_{f, \max }$ the fibre strength, $\tau_{s}$ the shear yield strength of the glue and $D$ the fibre diameter. For carbon fibre and realistic matrix shear yield strengths, the length $L$ amounts to a few hundred micrometres. While this effect can be neglected for gauge lengths above $10 \mathrm{~mm}$, it may play an important role at shorter gauge lengths. At those lengths, the probability of fibre fracture in the stress build-up region needs to be accounted for. Phoenix and Sexsmith [74] have proposed such a theory. Yao and Yu [71] mentioned that the error introduced by ignoring the stress build-up region was around $5 \%$ in their experiments. This means that their gauge length of $40 \mathrm{~mm}$ should be increased to $42 \mathrm{~mm}$, and that their tensile modulus and failure strain would be underestimated by $5 \%$.

One way to avoid clamping effects would be to disregard specimens that failed in the tabs. This approach has two vital drawbacks. Firstly, it is not always possible to determine where fracture initiated, as multiple fragments may be produced. It may be possible to apply grease to the fibre to dampen the energy release upon failure, but this approach is not widely used. Secondly, the weak fibres are less likely to be disregarded than the strong fibres [50]. A weak fibre has a relatively large flaw, which makes that fibre more likely to fail within the gauge length instead of in the tabs. This would introduce systematic errors into the Weibull distribution. 


\section{Number of tests}

The number of tests to extract Weibull parameters typically ranges from 20 to 50 [46,52,7679], while only in a few studies sample sizes of 100 or more were used [44,45,51]. Thomason illustrated that a low number of tests may falsely lead to the conclusion of more than one flaw population [30].

Similarly, Berger and Jeulin [47] reported issues with the number of required tests. They proved that 30 tests are insufficient to accurately determine Weibull modulus and scale parameter [47]. Swolfs et al. [20] investigated the influence of Weibull parameters on the composite strength. Based on a Weibull modulus of 6 and a scale parameter of $3500 \mathrm{MPa}$, a certain number of strengths were virtually generated. The Weibull parameters were fitted to these virtual strength values using the maximum likelihood estimators. Figure 4 plots the result of the fitted parameters depending on how many virtual tests were performed. This reveals that a large number of tests are required to accurately determine the Weibull parameters. The determination of the Weibull modulus was found to be particularly troublesome, especially when taking into account that experimental errors were ignored.

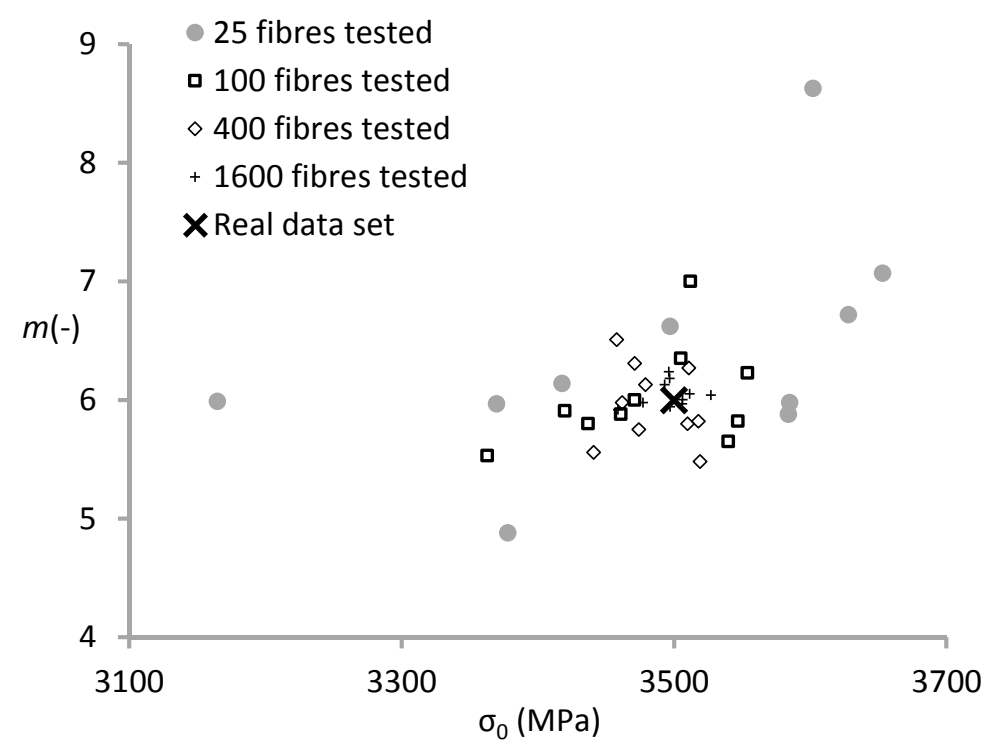

Figure 4. The estimates of the Weibull parameters based on 25, 100, 400 and 1600 virtual single fibre tests.

These virtual tests were generated from the real data set with $m=6$ and $\sigma_{0}=3500 \mathrm{MPa}$ (Adapted from [20] with permission from Elsevier).

Plugging the results of Figure 4 into a strength model revealed that several hundreds of single fibre tests were required to reduce the scatter in strength model predictions to less than $10 \%$. This means that even more tests are required when experimental errors are taken into account.

The studies described above all relied on the assumption of perfect measurements. Bermejo et al. [80] however, performed a similar statistical study, but included measurement errors. Bermejo et al. found that these measurement errors also lead to a statistical bias for smaller Weibull moduli. This makes sense, as the measurement errors introduce additional scatter, which widens the measured strength distribution and thereby decreases the estimated Weibull modulus (see Figure 1). For measurement errors of 5-10\%, this effect was strong, especially for Weibull moduli above 10. Luckily, the Weibull moduli for carbon fibres are typically in the range of 5 to $8[77,81,82]$. 
It is impossible to set up a generally applicable number of tests that should be performed. This depends strongly on the Weibull modulus of the fibre [83]. If the Weibull modulus can be estimated, then a simple statistical calculation such as the one in [20] can determine the maximum accuracy resulting from a given number of tests.

\section{Gauge length}

The gauge length is a crucial parameter in the Weibull distribution. The nature of the Weibull distribution permits an extrapolation towards other gauge lengths, using equation (4). There are however several reports in the literature claiming that this extrapolation is not accurate. Some of this work led to modified distributions, which were described in more detail in "2.1.2. Modified Weibull distributions".

Berger and Jeulin [47] performed an average of 30 single fibre tests on ceramic oxide fibres at six different gauge lengths $(5,10,25,50,100$ and $250 \mathrm{~mm})$. They found Weibull moduli ranging from 3.75 to 6.75 . They were able to explain these varying values based on statistical variation due to the limited number of tests. One can easily imagine that this may lead some researchers to wrongfully conclude that the Weibull distribution does not properly capture gauge length dependency.

Another key issue related to gauge length is the optimal gauge length for single fibre tests. As mentioned in "2.1.3. Experimental and statistical issues with single fibre testing", tests at gauge length below $10 \mathrm{~mm}$ may be affected by clamping and misalignment effects. Tests at large gauge lengths on the other hand, may increase the probability of preselecting the strongest fibres. From an experimental point of view, there may hence be an optimal gauge length. Even though this may be difficult to pinpoint, a $10-25 \mathrm{~mm}$ gauge length seems reasonable for most fibre types.

There may also be an optimal gauge length from a statistical point of view. This was recently investigated by Swolfs et al. [20]. The gauge length had no influence on the accuracy of the Weibull modulus estimate, whereas it did affect the accuracy of Weibull shape parameter estimate. To compare results at different gauge lengths, the results should be extrapolated to the same gauge length using equation (4). Since the exact Weibull modulus is not known, the estimated value has to be used, which introduces additional scatter in the data. The conclusion therefore was that the Weibull parameters should be measured at the gauge length that will be used. In the case of most strength models, this would imply that tests should be performed at gauge lengths well below $1 \mathrm{~mm}$. In this length regime, single fibre tests would not be reliable anymore due to the previously described clamping effects.

The importance of measuring at the relevant gauge length can be further illustrated with an example. Swolfs et al. [20] showed that the Weibull modulus of 6 could be determined with an accuracy of \pm 1 if about 100 tests were performed (see Figure 4). If these tests were performed at a gauge length of $10 \mathrm{~mm}$, then most models will require extrapolation down to much shorter gauge lengths. For a unimodal Weibull distribution, this extrapolation can be performed using equation (4). The results are plotted in Figure 5, revealing that the error in the Weibull scale parameter becomes very large for gauge lengths below $100 \mu \mathrm{m}$. Since Figure 5 does not even include experimental errors, it proves the importance of measuring the Weibull distribution at short gauge lengths. 


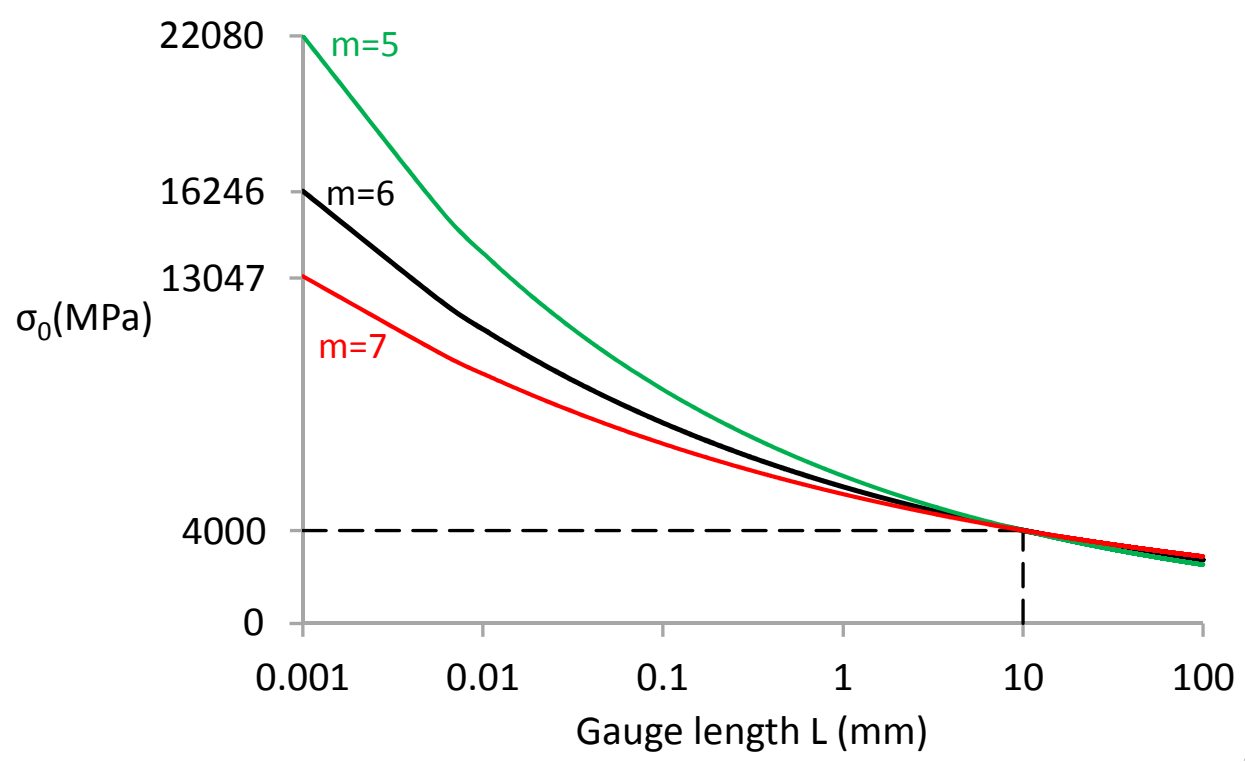

Figure 5. Variations in the Weibull modulus of \pm 1 lead to large errors at short gauge lengths. Results were calculated using a unimodal Weibull distribution with $\sigma_{0}=4000 \mathrm{MPa}$ and extrapolated using equation 4.

Another crucial implication is that performing single fibre tests at multiple gauge lengths without testing more fibres does not yield the benefit that is claimed by several authors $[40,60,84]$. Instead, it forces an extrapolation of at least part of the data, which introduces additional errors.

\section{Fibre cross-sectional variation}

Single fibre strength is often measured by determining the load at failure, and dividing this value by the average fibre diameter. Many authors have mentioned that this may not be a valid assumption [41,44-49,83]. Yu and Yao [45] for example found carbon fibre diameters ranging from 6.6 till $7.7 \mu \mathrm{m}$, while the nominal diameter was $7 \mu \mathrm{m}$. The average strength of the $6.6 \mu \mathrm{m}$ fibres was $3750 \mathrm{MPa}$, whereas that of the $7.7 \mu \mathrm{m}$ fibres was $2750 \mathrm{MPa}$. $\mathrm{Yu}$ and Yao noted that the size effect on fibre strength is actually much larger in the radial direction than in the length direction. This is seemingly in contrast with the results of Lara-Curzio and Russ [85], who proved that there was no correlation between diameter and strength. The reason for this apparent contradiction may be that $\mathrm{Yu}$ and Yao studied carbon fibres, whereas Lara-Curzio and Russ studied silicon carbide fibres. The failure micromechanisms and flaws may be different in both fibre types, although it is unclear how they would differ exactly.

Several standards for single fibre testing are in use, such as ASTM D3379-75, ASTM C155703 and BS ISO 11566-1996. It should be noted ASTM D3379-75 recommended using the average fibre cross-sectional area for converting failure load into strength. For the reasons mentioned above, this standard was withdrawn in 1998. The newer ASTM standard C1557-03 explicitly recommends using the individual fibre diameter instead of the average value. The BS ISO 11566-1996 standard does not require measuring the diameter of each individual fibre, but only recommends it if "the cross-sectional area of the fibres is known to vary widely". Unfortunately, variations of less than $10 \%$ in diameter can already cause large deviations $[45,86]$.

Most Weibull data in the literature has been measured using either the average or the nominal cross-sectional area. Measuring the diameter of each fibre is a tedious procedure, and is typically performed using either optical microscopy, scanning electron microscopy or laser diffraction. For optical microscopy, it should be noted that the wave length of the light limits 
the resolution to about $500 \mathrm{~nm}$. This is not accurate enough for most reinforcement fibres in composite applications.

Lara-Curzio and Russ [86] revealed that using the average diameter instead of the actual one leads to significant underestimations on the Weibull modulus. For a $1 \mu \mathrm{m}$ standard deviation on a mean diameter of $12 \mu \mathrm{m}$, a Weibull modulus of 4 would be measured if the actual Weibull modulus was 5. Taking into account the results in Figure 4 and 5, such an error would lead to major errors in strength models.

\subsubsection{Other testing techniques}

Other testing techniques have been developed to remediate the problems with single fibre tests described in the previous subsections. These subsections revealed that the focus should be on measuring a large number of fibres at short gauge lengths. The requirement for testing a large number of fibres also necessitates the need for a technique that is either fast or allows multiple measurements in a single test. Here, three techniques are proposed that incorporate these ideas as much as possible. Other techniques have been proposed in the literature, but are not as promising in light of the three requirements for an ideal testing technique. For example, the fibre bundle test $[46,50]$ was left out in the current review, as it cannot capture effects of fibre cross-sectional variation and is limited to relatively large gauge lengths.

\section{Fragmentation test}

The fragmentation test was originally developed for studying stress recovery in a broken fibre. This led to useful insights into the interfacial properties, which is required as input data for certain models [87-91]. Essentially, this test loads a single fibre composite, and monitors the number of fibre breaks as a function of the applied load. The fibre breaks are typically recorded through the use of polarised microscopy, as they cause gaps in the polarised light patterns (see Figure 6).

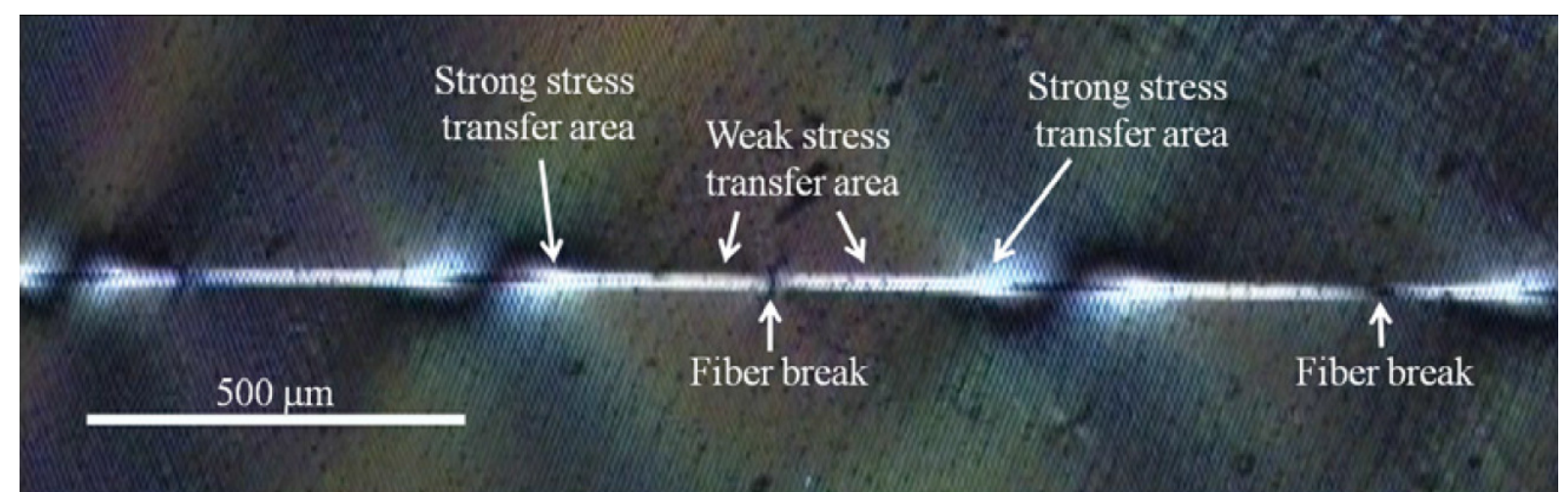

Figure 6. Typical fragmentation patterns of a T300 carbon fibre with 2 fibre breaks, revealing birefringence around the fibre breaks (Adapted from [92] with permission from Elsevier).

The test has also been used to determine the Weibull distribution for fibre strength [50,51,63,87,92,93]. Andersons et al. [50], Lachman et al. [92] and Shioya and Takaku [87] used a simple linear regression technique for calculating the Weibull distribution from the observed number of breaks as a function of applied strain. This approach is straightforward to apply, but can only be used when the number of fibre breaks is small compared to the length of the model. This means that the data at higher load levels cannot be used.

Both Hui et al. [94] and Zhao et al. [93] mitigated this drawback by applying the global load sharing theory (see "3.2. Load sharing rules" for further details on the different load sharing 
rules). This allowed them to incorporate the influence of the interactions between the fibre breaks. Watanabe et al. [51,63] recently improved upon this by using the shear-lag model of Okabe and Takeda [95]. The advantage of the approach of Watanabe et al., Hui et al. and Zhao et al. is that they can use all the observed fibre breaks, while the linear regression technique can only use the initial fibre breaks. For the same number of fragmentation tests, they can use more data points for fitting the Weibull parameters.

This approach has several key advantages over single fibre tests. Firstly, the test allows many data points to be gathered in a single test. On one hand, Watanabe et al. incorporated five fibres at a sufficient distance from each other. On the other hand, each sample breaks multiple times along its length. This led to about 50 data points for each test. Secondly, the nature of the fragmentation test allows measuring the Weibull distribution at gauge lengths that are not accessible by single fibre tests. Finally, the nature of this test permits testing of fibres inside a resin. If this resin has any influence on the Weibull distribution, then this test takes it into account. It should be noted however that such influence is highly speculative and has not been proven yet [22,92].

The fragmentation test for measuring Weibull distributions also has three drawbacks. Firstly, when the samples are cured, a compressive stress is exerted onto the fibre(s) [96]. This stress can be taken into account in the calculations, but care should be taken that it does not cause undulations in the fibre. Secondly, the data reduction scheme of Watanabe et al. [51,63] requires a model to fit the Weibull distribution. Requiring a model to measure modelling input data may not be the most robust method. Nevertheless, Watanabe et al. [63] found that the agreement with single fibre test data was excellent at long gauge lengths. Finally, there is a lower limit to the gauge lengths for which information can be obtained. A strong fibre/matrix interface is required to favor fibre failure over fibre/matrix failure [97]. This approach is hence not suitable for all possible fibre/matrix combinations.

\section{Loop test}

The second test is the loop test. This was originally developed in 1950 by Sinclair [98] to measure both the modulus and strength of glass fibres (see Figure 7). A loop is made in a single fibre, and this loop is confined in a single plane by two glass plates. A lubricant is added between the glass plates to minimise friction. The two ends are then pulled apart, which reduces the radius of curvature of the loop. Microscopy is typically used to measure the radius of curvature at the top of the loop. Fukuda et al. [99] later developed mechanics equations to calculate this radius of curvature based on the displacement of the loop ends. Combining the radius of curvature with the elastic modulus and fibre diameter is sufficient to calculate the stresses in the fibre. The last radius of curvature prior to final failure can hence be used to calculate the fibre strength.

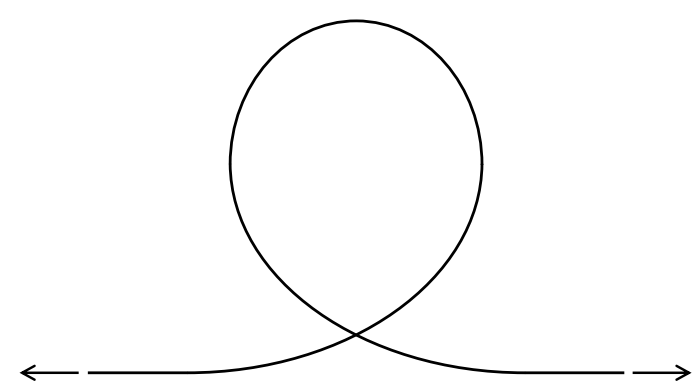

Figure 7. Illustration of the loop test, where the two fibre ends are pulled apart to increase the stress at the loop tip. 
Sinclair noted that the measured strengths were two to three times higher than in conventional tests. This was attributed to a lower probability of finding a flaw at the loop tip, where the stress is at its maximum. While this may seem like a logical conclusion now, it should be noted that Sinclair published his findings in 1950, which is one year before Weibull published his seminal work describing the Weibull distribution [5].

As mentioned by Williams et al. [100], the equations for a loop of an anisotropic material differ from those for an isotropic material. The low shear modulus of carbon fibre can influence the shape of the loop. Furthermore, the equations of Fukuda et al. [99] were derived assuming linear elasticity. This may introduce errors, as carbon fibre and several other fibres are not linear elastic. In the case of carbon fibre for example, an increase of $20 \%$ in stiffness is expected for every $1 \%$ of applied strain [101,102].

An important drawback of the loop test is that the stress is not uniform. The varying stress along the fibre length hampers the assignment of a gauge length to the measured fibre strength [103]. This stress variation over the cross-section makes the loop test more sensitive to surface flaws than a single fibre test. This may imply that a one-to-one correlation with single fibre test results does not exist. This inherent assumption in the loop test may be difficult to verify.

While the loop test can be useful to measure fibre strength at short gauge lengths, it does not address the issue of the number of tests. This is a relatively slow and tedious tests requiring careful sample preparation. Testing more than a 100 fibres will be extremely time-consuming.

\section{Synchrotron radiation computed tomography on UD composites}

The third test is synchrotron computed tomography (CT) on UD composites. By combining many projections, a 3D visualisation of the composite can be generated. Several thousands of fibres can be loaded in single specimen and individual fibre breaks can be visualised as a function of the applied load. Using suitable fibre break detection algorithms, this leads to a plethora of data on fibre breaks as a function of the applied load. To avoid the drawback of requiring a model for data reduction, the CT measurements should focus on low load levels to ensure a negligible number of fibre break clusters. This condition is required to allow a direct fit of the Weibull distribution and to avoid the use of a model for data reduction. It should be noted though that CT measurements on UD composites so far focused on high load levels $[17,18,22]$. Using an advanced strength model, it may even be possible to fit the Weibull parameters in such a way that the fibre break predictions match the experimentally found fibre breaks. First attempts have however shown that this is very challenging [22].

The advantage of synchrotron CT lies in its capacity to perform a huge number of simultaneous measurements at relatively short gauge lengths. The disadvantage is its limited availability, which is why it cannot be used for routine measurements. Nevertheless, it could have great value in the initial developments and as comparison for data coming from other testing techniques.

\subsubsection{Conclusion}

The Weibull distribution for fibre strength is the most important input parameter for strength models. Despite many detailed investigations by different researchers, measuring the Weibull distributions remains particularly difficult. The literature has not yet agreed on the most suitable distribution, nor on the best testing technique. This may also depend on the fibre type 
or on the purpose for which the data are used. Clearly, more research is needed in this area, as these input data are crucial for any strength model for UD composites.

Some authors believe that the Weibull parameters differ from one batch or bobbin to another. Given all the experimental and statistical issues described in this section, it seems highly unlikely that this has ever been proven with statistical significance.

\subsection{Matrix and interfacial properties}

The second key aspect in any strength model is the stress redistribution around fibre breaks. When a fibre breaks, the surrounding matrix is loaded in shear and helps to recover the stress in the broken fibre. This leads to a reduced stress over part of the length of the broken fibre, and an increased stress in the nearby fibres. These stresses are often described relative to the nominal stress level, in which case they are called stress concentration factors (SCFs). The magnitude of the stress concentrations and the length over which they are significant is controlled by the matrix and fibre-matrix interfacial properties. The fibre stiffness also plays a role, but this is often investigated using the ratio of fibre over matrix stiffness. This review will thus not deal separately with fibre stiffness. There is a long list of parameters that may affect the stress redistribution, but most models only use a few of them. The relevant parameters will be described in this subsection.

Most matrix systems are not linearly elastic, but visco-plastic materials. This results in additional complexity in most strength models, causing most researchers to simplify this behaviour, for example by treating the matrix as linear elastic or perfectly plastic. The question therefore rises in which cases these simplifications are justified. On top of that, recent experimental evidence revealed that the microscale resin properties can differ significantly from the macroscale properties that are commonly used. Finally, the fibre-matrix interface may also debond, introducing additional complexity. These aspects will be described in the next subsections.

The influence of thermal residual stresses will not be covered in the following sections. When thermoset resins are cured, the matrix shrinks and puts a compressive stress on the fibres. In polymer matrix composites, these effects are generally considered to be negligible [92,104,105]. As pointed out by Xia et al. [106], the residual stresses can be significant in metal matrix composites.

\subsubsection{Elasto-plastic materials}

If viscous contributions are ignored, then the matrix can often be considered as an elastoplastic material. Many authors have however used linearly elastic or perfectly plastic assumptions. The early works in this area focused on a single fibre, and analysed the stress redistribution using the shear-lag theory. The original model of Cox [107] assumed that the matrix cannot carry axial loads and that the interface does not debond. Extensions to multifibre composites were pioneered by Hedgepeth and Van Dyke [6,7], but these were still limited to linearly elastic materials. Later, shear-lag models were developed that included viscous [108,109] and plastic effects [95,110-112]. In the nineties, the improvements in computational power allowed finite element models to become a prominent tool for analysing stress redistributions [12,104,105,113-115].

Landis and McMeeking [110] created an excellent overview of the parameters influencing stress recovery of a broken fibre in a composite. Figure 8 displays the influence of various 
parameters in dimensionless terms, assuming that the fibre/matrix interface was well bonded. The stress was normalised by dividing by the applied strain and longitudinal fibre stiffness. The distance from the fibre break was normalised by dividing by the ineffective or slip length. The term slip length is often used when its value can be directly defined from the stress recovery profile. This is for example the case in the perfect plastic case (indicated by the dashed line in Figure 8). The other cases in Figure 8 however do not allow a straightforward measure for the stress recovery length. In that case, the term ineffective length seems to be more common. This length is typically defined as the length over which the stress is lower than $90 \%$ of its nominal value. It should be noted however that this definition is arbitrary, and is, just like the slip length, simply a measure for the length over which stress recovery occurs. This measure is crucial however, as it is closely linked to the extent over which the stress concentrations are of significant magnitude.

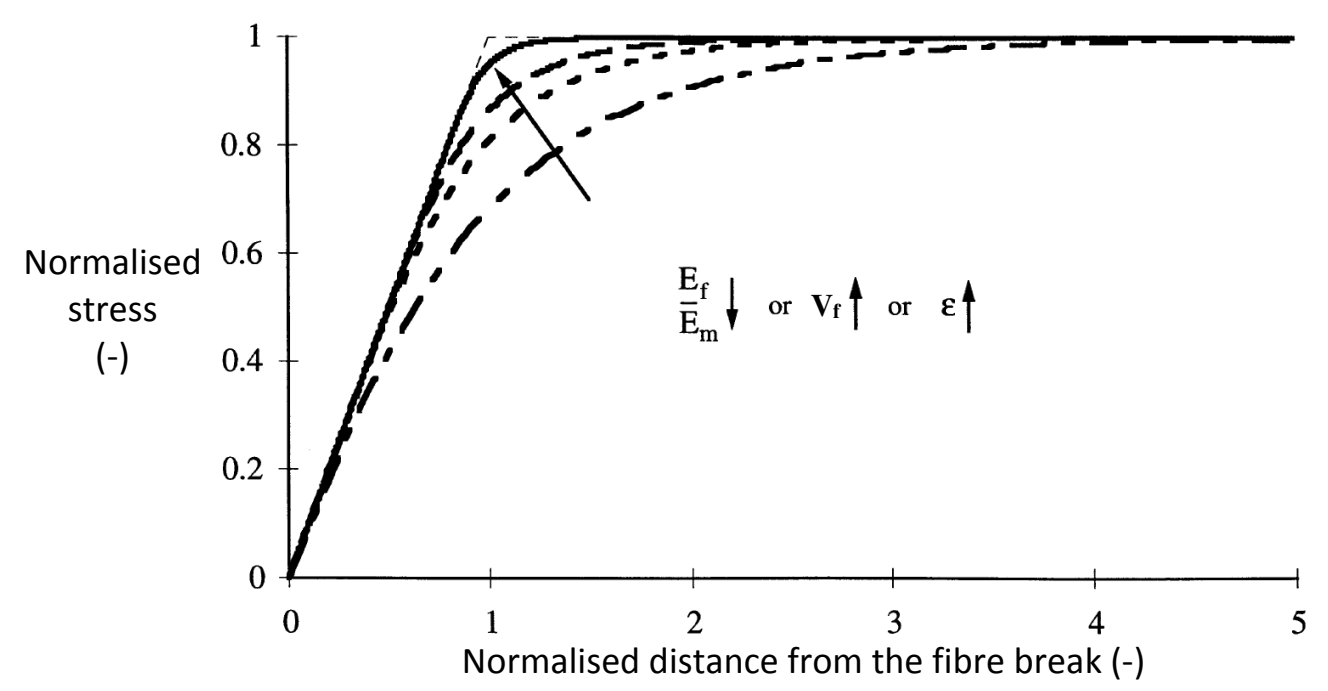

Figure 8. Overview of the influence of different parameters on the axial stress recovery in a broken fibre of a UD composite (Adapted from [110] with permission from Elsevier).

The model of Landis and McMeeking assumes linear elastic/perfect-plastic behaviour of the matrix. The reference case in this scenario is the solution for a perfectly plastic matrix, which leads to a bilinear stress recovery profile (see dashed line in Figure 8). A decrease in the ratio of fibre-to-matrix stiffness $E_{f} / E_{m}$ causes a closer approximation of the perfect-plastic behaviour. The normalisation removes the influence of any changes in the shear yield strength. The observed effect is thus attributed to an increase in the amount of plastic yielding, as the higher $E_{m}$ for a given $E_{f}$ causes the yielding onset to be reached earlier in strain terms. An increased fibre volume fraction $V_{f}$ and increased applied strain $\varepsilon$ have the same influence, as they also enlarge the plastic yielding region.

Other authors confirmed the trends that were described by Landis and McMeeking. The elastic FE model of Swolfs et al. [11] confirmed that a smaller fibre-to-matrix stiffness ratio $E_{f} / E_{m}$ reduced the ineffective length. Similarly, a decrease in the ineffective length was found with increased fibre volume fraction. This decrease essentially follows the same trend as the arrow in Figure 8, thereby confirming the results of Landis and McMeeking. An additional contributing factor was however suggested based on shear stress transfer in the intact fibres. The intact fibres increase the homogenised shear stiffness of the material around the broken fibre, thereby reducing the ineffective length. This effect is more pronounced at higher fibre volume fraction, as the homogenised shear stiffness would be higher. Such 
effects cannot be captured by most shear-lag models, as they normally ignore the shear stresses in the fibre.

In general, the yielding of the matrix reduces the efficiency of the load transfer, thereby smearing out the stress concentrations over more fibres and a larger length $[13,32,114,116,117]$. The smearing out decreases the probability for cluster development, whereas the larger length increases it. These counteracting effects make the influence on the strength difficult to assess. Behzadi et al. [32] predicted that a yielding matrix increased the composite strength by about $10 \%$ compared to an elastic matrix. The authors only modeled a single layer, and then extrapolated the results. This single layer supposedly had a thickness equal to the ineffective length, which was $149 \mu \mathrm{m}$. Despite the authors themselves showing that the ineffective length changed depending on the matrix behaviour, the layer thickness did not depend on the matrix properties. This may have affected the results.

De Morais [118] found that increasing the shear yield strength from $40 \mathrm{MPa}$ to $100 \mathrm{MPa}$ increased the tensile strength by about $25 \%$. This correlates well with the results of Pimenta and Pinho [34]. At first sight, this seems to contradict with the results of Behzadi et al., although it is difficult to make a direct comparison. Behzadi et al. compared a full elastoplastic matrix to an elastic matrix, whereas De Morais as well as Pimenta and Pinho changed the shear yield strength in a perfect plastic matrix. An additional difficulty in this comparison is that size effects may differ depending on the matrix behaviour. Okabe et al. [39] for example found that including the strain hardening in the plastic region significantly changed the size scaling of their predictions. Behzadi et al. [32] revealed a similar influence of size scaling.

Measuring the elasto-plastic response of a matrix material is not as straightforward as it may seem. In tension, thermoset resins may fracture at relatively low strains due to the presence of voids or other types of defects. Inside a composite, the presence of the fibres prevents these large defects from forming. This essentially means that a tensile test does not allow capturing the entire stress-strain diagram that is relevant for strength models. A common way to resolve this issue is to test the resin in compression $[13,119,120]$. This allows to reach much higher failure strains, thereby capturing the entire stress-strain diagram relevant for strength models. The main contribution of the matrix in the composite is however through shear stresses. Measuring the shear response is challenging, as it is difficult to achieve pure shear. The linear elastic shear response may be predicted from the tensile response if the matrix is linearly elastic. However, this assumption is only valid in some rare cases.

A potential problem when incorporating the full elasto-plastic matrix behaviour is that the stress redistribution will depend on the applied strain. This means that the stress redistribution around a single fibre break will change when the applied strain is increased. Implementing this continuous change is easy in some models [34,37,38], but computationally expensive in others [23,32]. This issue has been partially resolved by calculating the stress redistribution at a strain close to the failure strain of the composite $[23,32]$. This will reduce the accuracy of the predictions at lower strains, but the results should still be sufficiently accurate for most purposes.

\subsubsection{Microscale properties}

The literature has described in detail how the fibre strength depends on the length (see " 2.1 . Fibre strength"). The logical next question is whether the matrix properties also depend on the specimen size. Just like most reinforcement fibres, most thermoset resins are brittle. Resin 
failure is hence controlled by the presence of flaws, which inevitably leads to size effects $[121,122]$. The typical size of a resin pocket in fibre-reinforced composite depends on the fibre diameter, and ranges from hundreds of nanometres up to tens of micrometres [123]. Additionally, the large surface area of the fibres may also affect the nucleation of the resin curing. This could influence the degree of cross-linking. Nevertheless, the matrix properties used as input data for strength models are always measured on macroscale specimens.

Odom and Adams [124] tested neat epoxy dog-bone specimens in tension. They varied the cross-sectional area, but kept the gauge length constant. The tensile strength increased from $41 \mathrm{MPa}$ to $94 \mathrm{MPa}$ when the gauge volume decreased from 5 to $0.06 \mathrm{~cm}^{3}$. This strong increase was attributed to a decrease in flaw size from about $250 \mu \mathrm{m}$ to $100 \mu \mathrm{m}$. Odom and Adams correctly note that the presence of the fibres prevent such large flaw sizes from forming in a real composite. Other studies later confirmed these findings [122,125].

Hobbiebrunken et al. [126] went one step further. They devised a new manufacturing method to produce microscale epoxy fibres with diameters down to $22 \mu \mathrm{m}$. The fibres were drawn from the bulk polymer when the resin is vitrifying. Their tensile strengths were determined using the single fibre test described earlier ("2.1.3. Experimental and statistical issues"). Their results revealed a strong size scaling effect, with epoxy fibres of smaller diameters being significantly stronger (see Figure 9). The highest reported value was $166 \mathrm{MPa}$, which starts to approach the $275 \mathrm{MPa}$ theoretical strength of the epoxy used in their study.

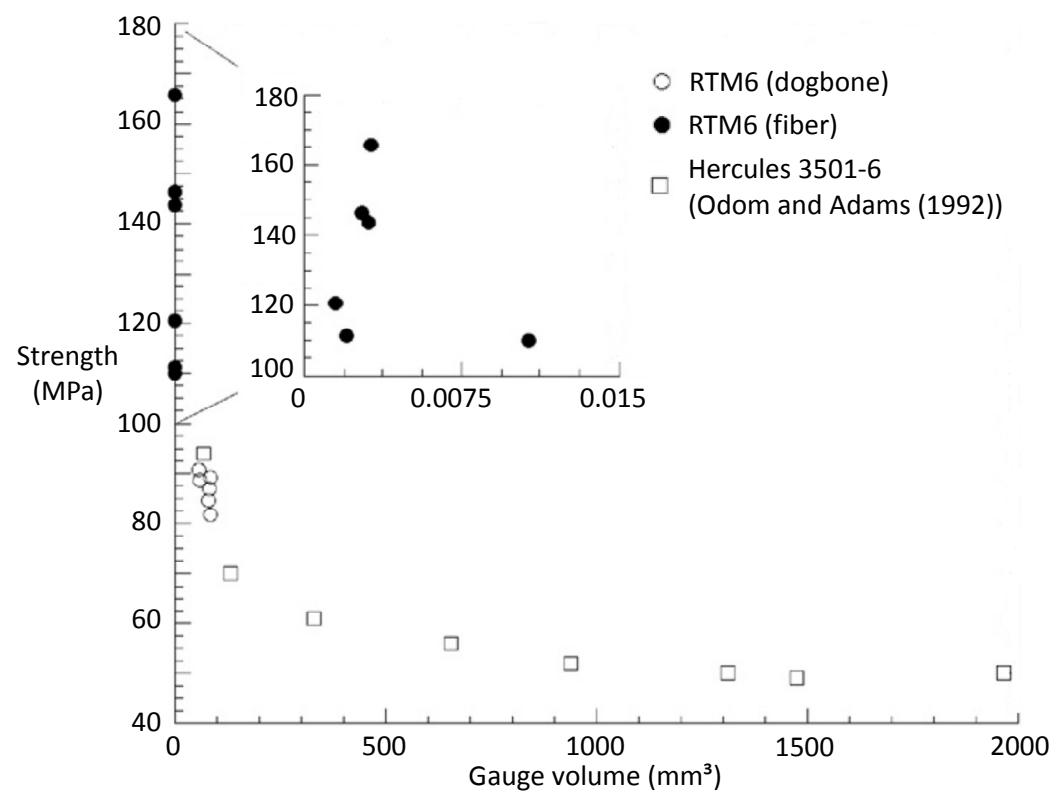

Figure 9. Tensile strength as a function of gauge volume. Some data on dogbone samples from Odom and Adams [124] are also included (Adapted from [126] with permission from Elsevier).

The drawback of the data of Hobbiebrunken et al. is that they focused solely on the tensile strength. Recently, new and more detailed results on microscale properties have become available. Misumi et al. [127] used an approach similar to Hobbiebrunken et al. to manufacture epoxy fibres. In contrast to their macroscale specimens showing brittle behaviour, their microscale specimens showed ductile yielding behaviour with failure strains ranging from $20 \%$ to $42 \%$.

The results of Misumi et al. [127] are thought-provoking and could have major implications on strength models. Nevertheless, three aspects would require further investigation. Firstly, 
Misumi et al. did not investigate how their manufacturing process affected the molecular alignment of the epoxy. Their epoxy fibres were drawn close to the point of vitrification. At this point, some but not all of the cross-links have been formed. It is therefore possible that some degree of orientation of the oligomers was frozen in during vitrification, and that this also had an influence on the cross-link density. If correct, then the high strength values may be attributed to molecular alignment and the yielding to a lower crosslinking density. Secondly, their epoxy fibres are mainly curing while hanging freely in air, whereas the presence of fibres would create nuclei for curing of the resin. This is likely to affect the crosslinking density, but there is no data in the literature to estimate the importance of this effect. Finally, to investigate the importance of microscale properties of the matrix for strength models, information on the tensile properties alone is insufficient. Shear properties on the microscale would be even more useful for strength models. Unfortunately, these data are not yet available in the literature.

\subsubsection{Time-dependent aspects}

Many matrix systems used in polymer and metal matrix composites are viscoelastic at their use temperature. This means that the constituent properties are time-dependent, which has three major consequences:

- A matrix that is under constant load will creep, thereby gradually reducing its stress and increasing the stress on the fibres [108,128].

- The stress recovery length of a broken fibre increases as a function of time, thereby causing the stress concentrations to extend over a larger length [108,129-133].

- The stress concentrations in the nearest neighboring fibres reduce, but increase on the fibres that are further away $[130,131,134]$. Essentially, this is equivalent to a matrix that becomes more ductile with time. As described in "2.2.1. Elasto-plastic materials", a more ductile matrix smears out the stress concentrations over more fibres.

This has several implications on strength models. Firstly, fibre-reinforced composites may creep at constant load [108,109,132,135]. Secondly, fibre breaks can continue to develop when a composite specimen is held at constant load [136]. This may be important for computed tomography studies where specimens are typically held at constant load during each measurement $[18,22]$. Finally, the strength of a composite is rate-dependent $[137,138]$. This may not be relevant in standardised tensile testing, but could be important for practical applications.

A landmark study of creep in fibre-reinforced composites was performed by Lifshitz and Rotem [132]. They tested UD glass-fibre reinforced composites, where one had a brittle, high modulus epoxy matrix and the other one a ductile, low modulus polyester matrix. The lowest constant load that was sufficient to cause creep failure in the brittle matrix composite was 0.72 times the strength in a quasi-static tensile test. For the ductile matrix composite, this reduced to 0.5 times the strength. These tests took 44000 and $33000 \mathrm{~min}$ respectively, which corresponds to about a month.

Du and McMeeking [108] developed a global load sharing analysis (see "3.2. Load sharing rules") for creep of metal matrix composites. Just like McLean's earlier work [139], this included creep of the matrix, which occurs even in the absence of fibre breaks. Especially in metal matrix composites, this can significantly increase the stress on the fibres. Du and McMeeking [108] however extended McLean's model to incorporate the increase of the ineffective length with time. They found that this increase had a strong effect on the creep 
rupture time at low stress levels. At high stress levels however, the ineffective length increase had a negligible influence on the predictions.

Chou et al. [136] found that the occurrence of large fibre break clusters was much more common under sustained loading than under monotonic loading. This finding is in line with Beyerlein et al. [134], who proved that the effect of creep is sped up when multiple fibre breaks interact.

Foreman et al. [137] proved that increased strain rates lead to slightly shorter ineffective lengths, but slightly larger stress concentrations. At higher strain rates, the polymer matrix behaves stiffer, causing a localisation of the stresses both in the fibre break plane and along the fibres. This led to slightly lower strengths at high strain rates, with the difference becoming larger for longer specimens.

For most applications, neglecting the time-dependent aspects is likely to be a reasonable assumption. In some specific cases however, these aspects should be taken into account. Care should be taken whenever samples are held at constant load or at elevated temperatures. One of the key difficulties seems to be to obtain reliable data of the time-dependent properties of the matrix. Most models have used a power law creep of the matrix [108,109,129,130,134,135,139], although some used more advanced laws [137]. The timedependent issues described here are mainly relevant for metal matrix composites, as they are often used at high temperature that allow the metal matrix to creep.

\subsubsection{Interfacial properties}

When a fibre breaks, large shear stresses arise in the matrix and at the fibre-matrix interface. This can cause the interface to debond, thereby increasing the ineffective length. This is often observed in single fibre composite fragmentation tests [140-143]. The debonding is controlled by parameters such as the interfacial shear strength, the strain energy release rate, the fibrematrix friction, the matrix yield strength and the fibre stiffness. Depending on the nature of the model, some of these parameters may not be needed. Many strength models in the literature even ignore debonding altogether.

To the best of our knowledge, in situ observations of interfacial debonding do not exist for composites with thousands or millions of fibres. Even in detailed synchrotron radiation computed tomography experiments, the resolution is insufficient to see fibre/matrix debonding $[17,18,21,22]$. The two debonded surfaces stay in close contact, making it impossible to detect them with computed tomography. Most other techniques such as microRaman spectroscopy or polarised light are limited to surface measurements. It is therefore unknown whether the knowledge from micro-composites with only a few fibres can be reliably transferred to macro-composites with millions of fibres.

The interfacial shear strength is an important parameter for the strength of UD composites. A low interfacial shear strength results in longer ineffective or slip lengths, which means the stress concentrations are significant over a larger region. This results in poor composite tensile properties. A strong adhesion however can also be detrimental, as it tends to localise the fractures in the same plane. Therefore, the general belief is that an intermediate interfacial strength leads to the best tensile properties [143-145].

Various methods can be used to reliably measure the interfacial shear strength. Several excellent reviews of these methods have been published in the past [146-148]. The single 
fibre fragmentation test [143,149], microdroplet/microbond test [150,151], single fibre pullout test $[152,153]$ and push-out test $[154,155]$ are the most common ones. These four methods are schematically depicted in Figure 10. The advantage of the push-out and pull-out test over the fragmentation test is that they can also yield a value for the friction coefficient. This parameter is also important, as it is required for modelling stress redistributions with interfacial debonding. However, as mentioned by Zhandarov and Mäder, the fragmentation test is more relevant for composites with brittle fibres and ductile matrices. In all techniques, the data reduction should be done with care, as there are many different reduction schemes. A detailed list of the advantages and disadvantages of the various methods can be found in the review of Herrera-Franco and Drzal [147].

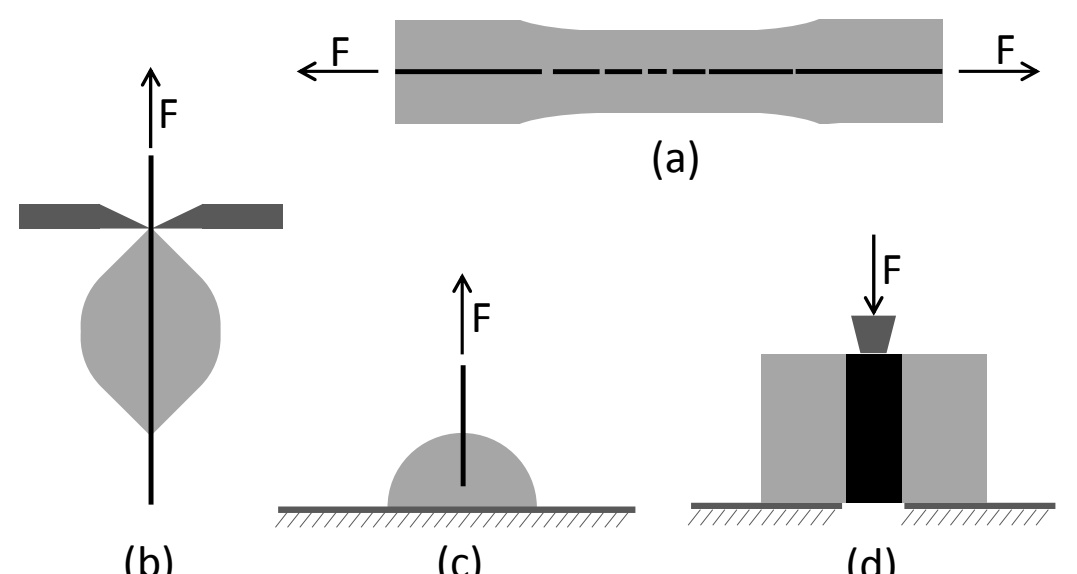

(b)

(c)

(d)

Figure 10. Schematic illustration of the four most common techniques for measuring interfacial shear strength: (a) single fibre fragmentation test; (b) microdroplet/microbond test; (c) single fibre pull-out test; (d) push-out test. Black indicates the fibre, light gray the matrix and dark gray the supports or auxiliary materials. The arrows indicate the direction of the applied force $F$.

Zhao and Takeda [156] developed a strength model that takes into account interfacial debonding. In general, they found good agreement with experimental data, but not when the interfacial shear strength was high. For the high interfacial shear strength case, it was hypothesised that the interface did not debond, but the matrix cracked instead. Recent modelling evidence has however revealed that local matrix cracking around fibre breaks has a negligible influence on the longitudinal strength of UD polymer matrix composites [23]. Its influence in metal or ceramic matrix composites is likely to be higher due to their higher matrix stiffness.

Xia et al. [106] proved that low friction causes a large ineffective length, and hence that the stress concentrations are of a significant magnitude over larger distance from the fibre break. $\mathrm{Xia}$ et al. also noted that low friction reduces the stress concentration, and attributed to the increasing contribution of the matrix in the load transfer. This was the case in a metal matrix composite, so the lower matrix modulus in a polymer composite is likely to reduce this effect. Increasing the friction coefficient from 0.25 to 0.9 increased the tensile strength by only $3 \%$ [106]. Van den heuvel et al. [105] reported that a friction coefficient in the range of 0.1 to 0.4 is realistic for carbon fibre/epoxy composites. This conclusion was based on a comparison of Raman spectroscopy with a finite element model. The friction coefficient is therefore unlikely to have a major effect on the tensile strength of polymer matrix composites.

An important remark with respect to friction is that none of the published works using a frictional coefficient relied on direct experimental measurements. Friction was taken into 
account either by trying out different values for the frictional coefficient [104-106] or by using an indirect and constant value for the interfacial sliding stress [156]. None of the existing works deals with the energy release rate for interfacial debonding or with variations in the interfacial bonding. Obtaining reliable measurements of the relevant parameters is clearly a challenging task.

\subsubsection{Conclusion}

While often considered less important than fibre strength, the matrix and interfacial properties can also have a major influence on the outcome of strength models. Measuring the required matrix and interfacial properties is often not trivial, and has received significantly less attention than fibre strength. Depending on the modelling assumptions, many different parameters may be needed and others may be obsolete. Table 1 summarises which models have used which type of properties. It should be noted that two of these parameters, the microscale properties and the strain energy release rate for debonding, have never been used in strength models. It is therefore impossible to judge their importance.

Table 1: Overview the matrix and interfacial properties that are relevant for strength models for UD composites, and where they have been used in the literature. References were only included if they provided modeling predictions.

\begin{tabular}{cc}
\hline Property & Used in references \\
\hline Elastic matrix only & {$[6,7,11,12,16,20,22,36,107,113-115,157-159]$} \\
Elastic/perfectly plastic matrix & {$[104,110,112,114,118,160]$} \\
Perfectly plastic matrix & {$[6,34,75]$} \\
Elastic/linearly plastic matrix & {$[95]$} \\
Entire tensile diagram of matrix & {$[23,104-106,111]$} \\
Entire compression diagram of matrix & {$[13,32,104,105]$} \\
Entire shear diagram of matrix & {$[33,137]$} \\
Residual stresses & {$[104-106]$} \\
Microscale properties & Nowhere \\
Viscoelastic properties & {$[33,108,109,128,129,133,137]$} \\
Constant interfacial shear strength & {$[145,156,158]$} \\
Strain energy release rate for debonding & Nowhere \\
Constant friction coefficient & {$[104-106,156]$} \\
\hline
\end{tabular}

\subsection{Conclusion}

The input data of any model are crucial for the outcome of the predictions. This is particularly relevant for strength models for UD composites, where the input data are often difficult to measure experimentally. The reliability of strength models can be greatly increased by improved test procedures for measuring fibre, matrix and interfacial properties. The above review of these parameters has pointed out which parameters have a large influence and which ones do not. This needs to be approached carefully though, as every fibre/matrix system is different.

\section{Modelling assumptions}

Apart from the input data, every strength model is based on a set of assumptions. This section will highlight four key assumptions in detail. These are related to fibre packings, load sharing 
rules, dynamic stress concentrations and multiple fibre breaks. Finally, section "3.5. Other assumptions" highlights a few smaller assumptions that have received little or no attention in the literature. This also implies that it will be more difficult to judge their importance.

\subsection{Fibre packings}

Many models in the sixties, seventies and eighties used 1D packings (see Figure 11a and b) to calculate the stress concentration factors (SCFs) on the intact fibres near fibre breaks [7,10,157-160] These SCFs were calculated as the relative increase in stress compared to the nominal stress level in the fibres. Assuming a single row of fibres greatly simplified the analytical equations, which led to the successful prediction of some of the basic effects present in UD composites. Nevertheless, a packing that has just two nearest neighbors for each fibre cannot be expected to yield accurate results for real composites. Hedgepeth and Van Dyke [6] for example predicted a static stress concentration factor (SCF) of 33\% for a 1D packing (see Figure 11a) compared to $10.4 \%$ for a 2D hexagonal packing (see Figure 11c). Similar conclusions were reached by Van den Heuvel et al. by comparing the FE results for a 1D [105] and 2D packing [104]. Therefore, the focus in the past decades shifted towards 2D packings, as they are more representative of real UD composites. The literature has used either hexagonal (see Figure 11c) $[6,8,9,12,32,33,37,39,95,110,112,113,118,161,162]$, square (see Figure 11d) $[6,13,38,163,164]$ or random 2D (see Figure 11e) packings $[11,16,20,22,23,36,163,165,166]$.

(a) Regular 1D

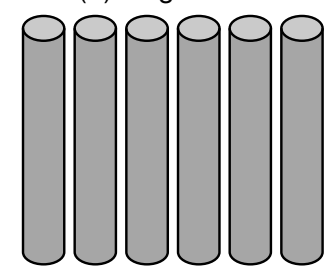

(c) Hexagonal 2D

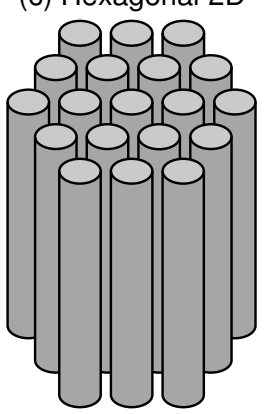

(b) Random 1D

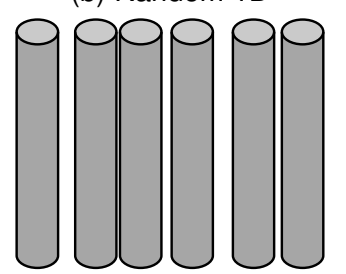

(d) Square $2 \mathrm{D}$
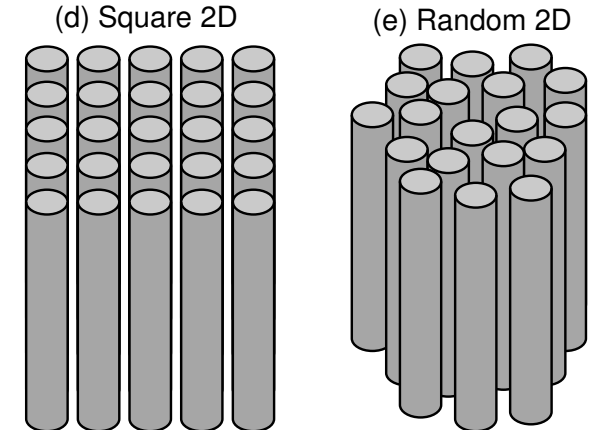

Figure 11. Illustration of the five different fibre packing types: (a) regular 1D; (b) random 1D; (c) hexagonal 2D; (d) square 2D; (e) random 2D.

Some comparative studies between the different 2D packing types have been performed. Landis and McMeeking [163] for example started off with a square packing, and distorted the packing by slightly moving one or more fibres. Moving all eight nearest neighbors closer to the broken fibre had only a minor effect on the SCFs. Moving just one fibre closer to the broken fibre increased the SCF on that broken fibre from $8 \%$ to $41 \%$. Curtin and Takeda [167] compared the strength predictions of a hexagonal packing to that of a square packing. Their simulations with a hexagonal packing lead to $1 \%$ higher strength than those with a square packing. 
An extensive finite element study of the influence of fibre packing was performed by Swolfs et al. [11,20]. Random packings were shown to lead to potentially much higher SCF values than regular packings [11]. This confirmed the findings of the shear-lag based predictions of Landis and McMeeking [163]. The observed SCF increases were however balanced out by other fibres having lower SCF values than in regular packings. A random fibre packing did seem to lead to shorter ineffective lengths than in regular packings. This effect alone would reduce the predicted strength, but may be counteracted by the higher SCFs. A strength model was therefore used to prove that the packing type results in variations of 3-4\% in the predicted failure strain [20]. These variations are smaller than the expected accuracy of strength models. In many cases, the use of 2D regular packings (see Figure 11c and d) is therefore justified.

Despite having little influence on longitudinal strength predictions for UD composites, random fibre packings should not be ignored completely. Firstly, random fibre packings are crucial for transverse strength predictions [168,169], but this is outside the scope of this review. Secondly, regular packings face issues when not all fibres have the same diameter. This would severely limit the maximum fibre volume fraction that can be achieved. Carbon fibres typically have limited diameter variations, and therefore do not face this issue. Natural fibre composites [170] and hybrid composites [36,171,172] would require random packings due to the large variations in fibre diameter.

\subsection{Load sharing rules}

Load sharing rules define how the stresses are redistributed when a fibre breaks. Many different rules have been implemented in the past, and can roughly be split up into four categories: equal, global, very local and local load sharing. Local load sharing means that the stress concentrations are spread not only over the nearest neighbors, but also over the fibres that are further away. The stress concentrations however decrease significantly when the fibres are further away.

Very local load sharing assumes that all stress concentrations are concentrated on the nearest neighbors only. This load sharing rule is often used in combination with regular packings, as the definition of nearest neighbors in a random fibre packing would be arbitrary. The very local load sharing rule was introduced by Harlow and Phoenix [62,173-175], and further developed by other authors [36,176-178]. Very local load sharing overestimates the localisation of the stress redistribution [179], but is nevertheless considered to be a reasonable approximation [177].

Global and equal load sharing spread the stress concentrations equally over all fibres present in the composite [162,180-185]. The difference between both lies in what happens away from the fibre break plane. In equal load sharing, the broken fibre is assumed to completely lose its load transfer capacity [180]. Essentially, this is equivalent to the situation in a dry fibre bundle. In global load sharing however, the broken fibre gradually recovers its stress as a function of the distance to the fibre break. This approach was initially developed by Curtin [186,187], and later extended by Neumeister [182,183] and Hui et al. [184]. In global load sharing, the influence of the other fibres is taken into account through their homogenised response. This leads to analytical solutions for the composite strength. Global load sharing rules can often provide a first indication of the importance of certain effects [188-190], after which a more refined study can be performed with more advanced load sharing rules. The global load sharing model has been particularly useful in ceramic and metal matrix composites [191,192]. 
There is no doubt in the literature that local load sharing is the most accurate rule for impregnated fibre-reinforced composites. The key question is however in which situations the other rules can be justified. The global load sharing approach has the advantage that it allows an analytical solution and hence a rapid scanning of the parameter space to find optimal performance $[188,189]$. Similarly, very local load sharing rules can be significantly easier to implement and more computationally efficient than local load sharing. Zhou and Curtin [193] proved that a more localised load sharing reduces the predictions of tensile strength. This means that equal/global load sharing leads to overpredictions of tensile strength, whereas very local load sharing leads to underpredictions compared to local load sharing. Another key implication of the load sharing rule is that local and very local load sharing can capture size scaling effects, whereas they are absent in global/equal load sharing analyses [193]. These effects should be considered when deciding which load sharing rule to use.

\subsection{Dynamic stress concentrations}

Intrinsically, fibre fracture is a dynamic process, resulting in a change in the stress level over time. This dynamic variation in stress is not that important for the broken fibre itself, but may be crucial for the nearby fibres. Prior to the fibre break, the stress in the nearby, intact fibres equals the nominal level (see Figure 12). When the fibre breaks however, the stress in the nearby fibres increases to the dynamic stress level, after which it gradually dampens out to the static stress level. The reason for these dynamic effects is that a broken fibre releases its stored elastic energy and converts it into kinetic energy. This causes dynamic stress concentrations on the neighboring fibres that exceed the static ones. Nevertheless, all state-ofthe-art strength models ignore the resulting dynamic instead of the static stress concentrations.

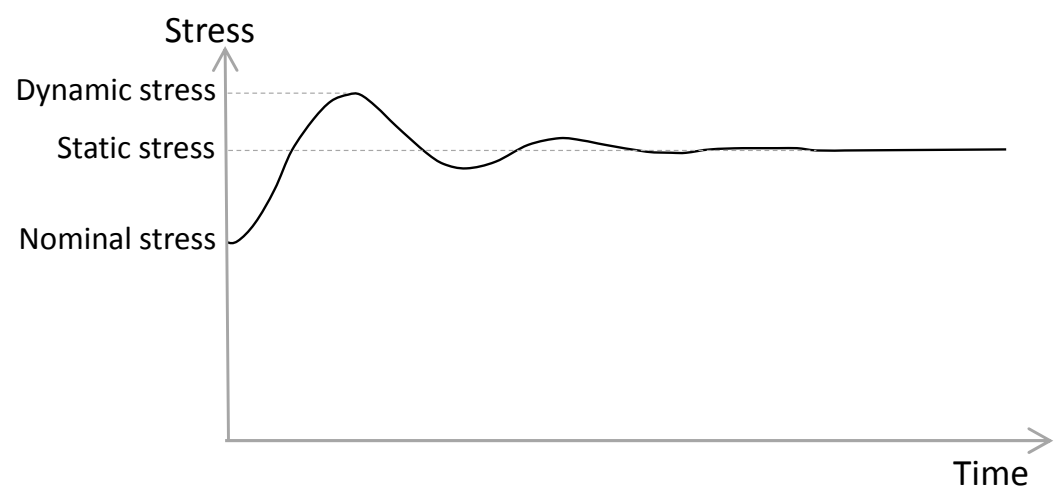

Figure 12. The stress evolution in a nearby, intact fibre as a function of time when a fibre breaks in a regular 1D packing. The dynamic stress level is significantly higher than the static stress level.

Experimental evidence indicated the importance of these dynamic effects. Van den Heuvel et al. [194] and Jones and DiBenedetto [195] both found that micro-composites tended to display coplanar fibre breaks. Such observation are unlikely to be explained based on statistical fibre strength and the static stress redistribution around a fibre break. Synchrotron radiation computed tomography measurements recently provided further indications that dynamic effects may be crucial in the development of clusters of fibre breaks [22]. These measurements revealed that fibre break clusters tended to develop in one load step, after which they did not seem to grow any further. This implies that further increasing the load was insufficient to break more fibres around the clusters. While direct evidence was absent, these observations may potentially be explained by large dynamic stress concentrations, happening at the moment of the first fracture. Additionally, a significantly higher ratio of coplanar to 
diffuse fibre breaks was found in experiments (see Figure 13) than in model predictions. Dynamic effects are the most reasonable explanation for such observations.
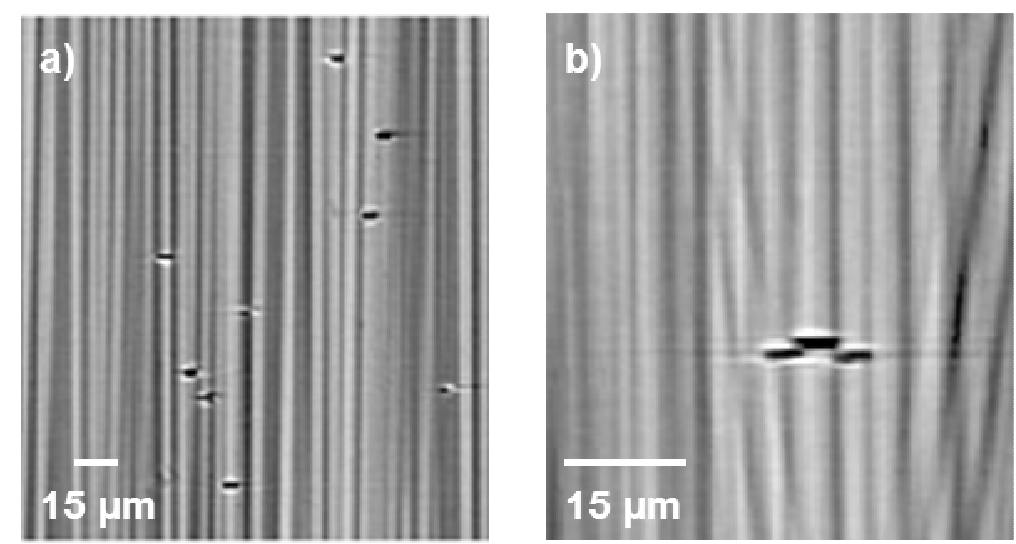

Figure 13. (a) Diffuse and (b) coplanar fibre breaks as measured by synchrotron radiation computed tomography (Adapted from [22] with permission from Elsevier).

The very first paper on stress concentrations in multi-fibre composites already included dynamic effects. Hedgepeth [7] modeled a regular 1D packing (see Figure 11a). For a single fibre break, Hedgepeth predicted a static SCF of $33 \%$ on the two nearest neighbors, whereas the dynamic one was $53 \%$. Hedgepeth reported this in terms of the dynamic response factor, which was the ratio of the maximum stress over the static stress. For a single fibre break, the dynamic response factor was $1.53 / 1.33=1.15$. This value looks rather low when calculated in this manner. In relative terms however, an SCF of 53\% is $60 \%$ higher than an SCF of $33 \%$. For predicting how much this increased the failure probability of the nearby fibres, the $60 \%$ is more relevant than the dynamic response factor.

Hedgepeth [7] also illustrated that the dynamic response factor increased from 1.15 to 1.19 and 1.20 for two and three simultaneous fibre breaks, respectively. This proves that dynamic effects could potentially be crucial to capture fibre break and cluster development in UD composites. The analysis of Hedgepeth however included several assumptions that may have increased the dynamic effects. Hedgepeth for example assumed a linear elastic matrix without axial load carrying capacity and ignored any viscous contributions. Furthermore, it is unclear how the dynamic effects in a 2D packing would differ from those in the 1D packing used by Hedgepeth.

Following the footsteps of Hedgepeth, some progress was made in the eighties. Ji et al. [196] used a similar approach, but also inspected the dynamic response factor along the fibre instead of just in the fibre break plane. This factor was ranged from 1.1 to 1.15 along the fibre, and slowly decreased with increasing distance from the fibre break plane. This decrease with distance would help to explain why coplanar fibre breaks are more common than diffuse fibre breaks (see Figure 13).

Sakharova and Ovchinskii published a series of three papers on dynamic effects in composites [197-199]. As evidenced from having no citations even after 30 years, these papers have remained relatively obscure to most researchers. Nevertheless, their model is relatively advanced, as it includes non-linear matrix behaviour and a 2D hexagonal packing. Sakharova and Ovchinskii [197,198] predicted dynamic SCFs that were about twice as high as the static SCFs, which is more than the $60 \%$ found by Hedgepeth [7]. They also found that the dynamic SCFs are the largest when the fibre volume fraction is 60-70\% [197,198]. This range is 
typical for an impregnated UD fibre bundle. Despite having the intrinsic capacity to study the influence of matrix non-linearities, Sakharova and Ovchinskii did not present any results on this matter.

Accorsi et al. [115] developed the first finite element model for dynamic stress concentrations. Their main motivation was to explain the strong "co-ordination of fibre breaks". This refers to works of Jones and Dibenedetto [195] and Van den heuvel et al. [194], who found that fibre breaks in micro-composites tend to occur within the same plane. This observation could not be explained based on static stress concentrations. Their finite element model was twodimensional and assumed plane strain conditions. Their dynamic calculations revealed that dynamic effects lead to an increased probability for these coordinated fibre breaks. They however did not calculate the influence of dynamic SCFs on the strength predictions.

There has also been some work on dynamic effects in hybrid composites. Xing et al. [200] modeled a composite consisting of a carbon and a glass layer. They revealed that the stress waves propagating through such a hybrid composite are always smaller than in the corresponding low elongation composite. This was true irrespective of the fibre stiffnesses or diameters, as any difference would tend to cause out-of-phase stress waves that reduce each other. This could be a potential contributor to the hybrid effect, which is a synergetic effect that increases the failure strain of the low elongation fibre in hybrid composites [201].

The presence of dynamic effects is widely accepted, but, to the best of our knowledge, they have never been implemented in strength models. Some information on the magnitude of dynamic stress concentrations is present in the literature, but all these models used relatively simple assumptions. The importance of these dynamic effects is therefore unknown in the literature. If the dynamic response factor is as large as predicted by these models, then significantly lower strength predictions are inevitable.

\subsection{Multiple fibre breaks}

Most models for stress redistribution deal with the situation of a single fibre break. Many of the analytical models can easily be extended to multiple fibre breaks [6,7,34,160,202-204]. This is more difficult for models that use FE analysis. Since modelling all possible cluster configurations is impossible, there was a need for superposition rules. These rules will predict the stress redistribution around multiple fibre breaks based on the solutions for single fibre breaks [23,205-207].

Sastry, Beyerlein and Phoenix [205,206] were the first to develop a superposition rule for multiple, non-coplanar fibre breaks. They extended Kachanov's fracture mechanics approach $[208,209]$ for the interaction of multiple cracks in a homogeneous solid to fibre breaks in composites. Their initial "break influence superposition" [206] extended Hedgepeth's model on 1D packings with coplanar fibre breaks to non-coplanar fibre breaks. This rule was later extended to the "quadratic influence superposition" rule [205], which was both faster and more accurate than the break influence superposition rule. It is an elegant approach, which works well in combination with shear-lag models.

Zhou and Curtin [193] developed a lattice Green's function model. This function is basically a response function capturing the displacement due to an applied load. If the Green's function for a single fibre break is known, then calculating all fibre stresses around multiple fibre breaks is relatively straightforward. The initial ideas of Zhou and Curtin later evolved into the 
spring element model, which consists of a set of axial and transverse springs (see Figure 14) [37,38]. The axial springs represent fibres, whereas the transverse springs represent the shear stress transfer of the matrix. The stress redistribution around multiple fibre breaks is calculated automatically as the algorithm searches for the equilibrium state of the system of springs. This is a very elegant solution to calculate the stress redistribution around multiple fibre breaks.

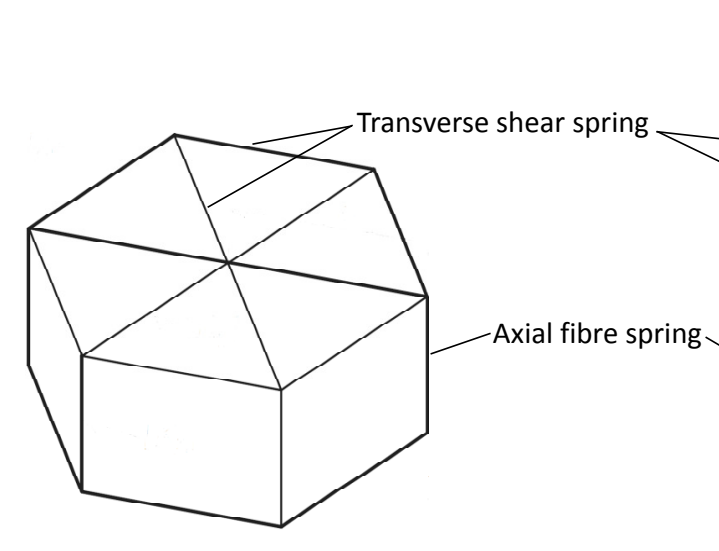

(a)

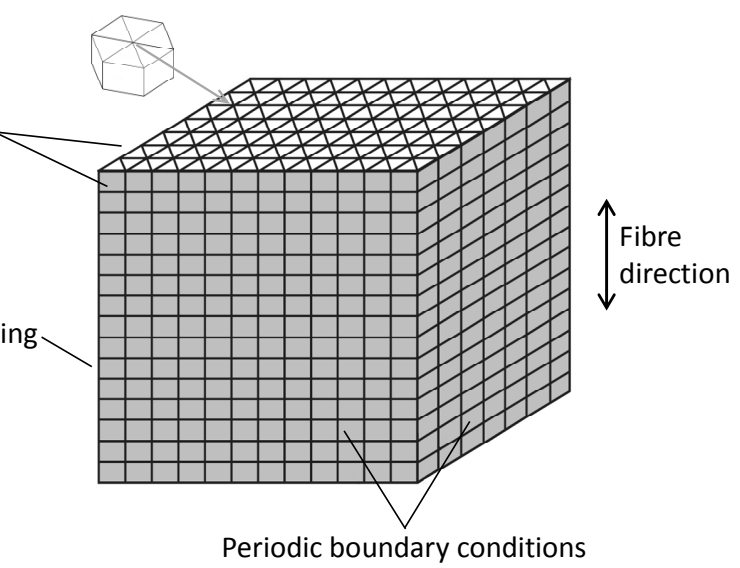

(b)

Figure 14. Schematic overview of the spring element model, where (a) the unit cell consists of axial fibre springs and transverse shear springs. The unit cells are assembled into (b) the full scale composite model (Adapted from [37] with permission from Elsevier).

Behzadi et al. [32] used a more ad-hoc approach by calculating the FE stress redistribution around clusters of two and three coplanar fibre breaks. The number of possible configurations is still manageable for these small clusters, but this number rapidly explodes for larger clusters. Unfortunately, it is not clear how Behzadi et al. dealt with larger clusters in their model. Dealing properly with those clusters is crucial for accurately predicting final failure of UD composites.

Swolfs et al. [23] proposed an approximate, but easy-to-implement method. The stress redistribution around multiple fibre breaks was approximated by a modified linear superposition of the single fibre break solutions. Linearly superposing these solutions would lead to underestimations of the stress concentrations, as this approach neglects the stress concentrations that broken fibres exert onto each other. A simple correction was proposed to resolve this, and its accuracy was validated using finite element models with three coplanar fibre breaks. The disadvantage of this approach is that it currently still ignores the increase in ineffective length with increasing cluster size. The literature currently does not reveal the importance of this effect.

\subsection{Other assumptions}

This section will review several less common assumptions that may influence the outcome of the models. Since they have received little attention in the literature, it is difficult to judge their importance.

\subsubsection{Stress variation of cross-section}

The stress redistribution around a fibre break does not cause a constant stress in the nearby fibres. Instead, the longitudinal stress in these fibres varies significantly over their crosssection, and is highest close to the fibre break [11-13,32,114]. An example is shown in Figure 
15 for a carbon fibre/epoxy composite with $70 \%$ fibre volume fraction. The nearby fibre was only $0.15 \mu \mathrm{m}$ away from the broken fibre, which caused stresses that were seven times higher than the nominal stress level.

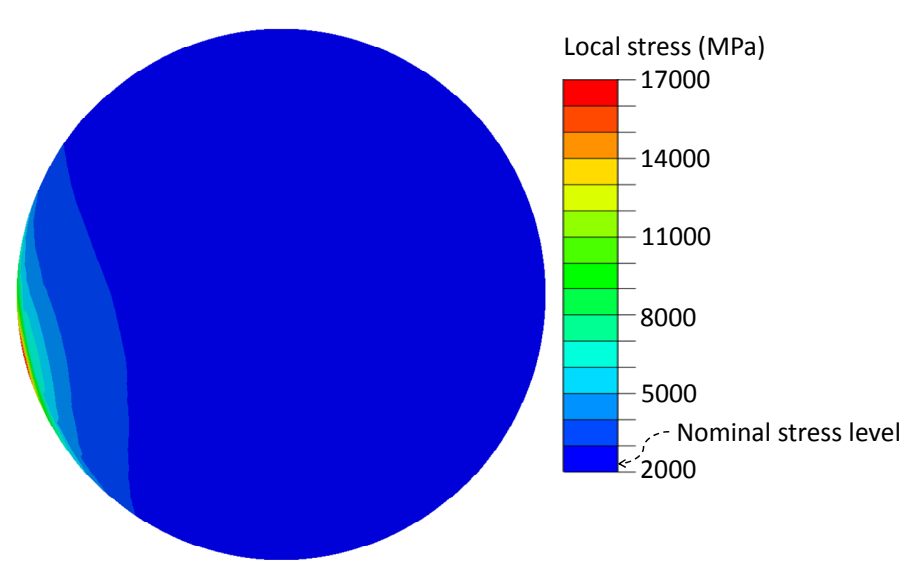

Figure 15. Local stress concentrations in the nearby fibres can be very high if they are close to the broken fibre. The maximum stress here is more than $\mathbf{7}$ high times higher than the nominal stress level, which was $2300 \mathrm{MPa}$. This calculation was performed for a carbon fibre composite with $70 \%$ fibre volume fraction and the shown fibre being $0.15 \mu \mathrm{m}$ from the broken fibre.

The stress variation of the cross-section has four implications. Firstly, the extraction of SCF values from FE models typically requires averaging the stress over the cross-section. Some authors have however analysed the stress at the surface or the stress in the centre line of the fibres [12,13,32,113]. Shear-lag models often assume that fibres are one-dimensional elements, and hence do not face this issue. Secondly, using the average stress will lead to different failure probabilities than integrating the local failure probabilities over the crosssection. This is because the Weibull distribution is not a linear function. Thirdly, since failure of carbon fibres is often dominated by surface flaws [53-55], the stress variation may increase the failure probability more than expected from the SCF averaged over the cross-section. This effect is currently ignored by all state-of-the-art strength models, as there is no Weibull strength distribution available at this scale. Finally, the stress concentrations may be so high locally that the theoretical strength of carbon fibre is exceeded. This strength is often assumed to be one tenth of the stiffness. In the case of carbon fibre, it is unclear which stiffness should be used in this calculation. It can either be the stiffness of the graphite planes (about 1000 $\mathrm{GPa}$ ) or the stiffness of the carbon fibre (typically 230-400 GPa).

The literature has given little to no attention to these implications. Their importance thus remains unknown.

\subsubsection{Planarity of fibre breaks}

Some models assume that fibre breaks occur in the same plane [32], even though the experimental evidence revealed that this is a simplification. Experimentally, about $50 \%$ of the fibre break clusters were found to be coplanar, whereas the others were diffuse (see Figure 13) [22]. Unfortunately, the literature does not offer any direct comparison to directly gauge the importance of this assumption. Based on the existing knowledge however, it is likely that this assumption has a major effect on the predicted strength. Existing models that do not require this assumption could be used to assess its importance. 


\subsubsection{Reduced defect sensitivity}

As mentioned in "2.1. Fibre strength", most Weibull distributions are measured using the single fibre test. In a composite however, the fibre is surrounded by matrix. The presence of the matrix might reduce the defect sensitivity of the fibre [22,92]. This hypothesis remains highly speculative, as direct evidence is completely absent. If a surface flaw is considered to be a tiny notch in the fibre, then this notch can be filled by matrix. This could potentially reduce the stress intensity at the notch tip, thereby increasing the strength of the fibre. If this hypothesis would be confirmed however, it would have major implications on the way fibre strength should be measured. Unfortunately, no one has investigated this problem yet.

\subsubsection{Critical cluster criterion}

Most strength models in the literature define final failure as the unstable propagation of a large cluster of fibre breaks. This unstable propagation is based on a stress analysis, whereas such a large "crack" may be controlled by strain energy release rate instead of stress considerations. Properly capturing this last part of the failure of UD composites may therefore require a fracture mechanics approach instead of a stress-based approach. Whether this is actually required depends on the size of the critical cluster, but relatively little is known on this size. The largest cluster that was observed experimentally consisted of 14 fibre breaks [18]. This was however observed at $94 \%$ of the failure load, and models have indicated the clusters rapidly grow in size in the last $5 \%$ of applied load [20].

Models that are fully based on fracture mechanics exist in the literature [210-213], but they often ignore many of the aspects described in this review. Models that combine the best of both worlds have not been developed yet.

\subsubsection{Fibre alignment}

Nearly all strength models for UD composites assume perfect fibre alignment. In real life, the fibres in UD composites are never perfectly aligned, and misalignment angles of a few degrees are unavoidable [214,215]. Although some models that incorporate fibre misalignment have been developed [166,216], they are scarce and have not been used to quantify the effect of the misalignments on strength predictions. It is therefore currently impossible to judge the effect of the fibre misalignment on the performance of UD composites.

\subsubsection{Non-linear elastic fibres}

The overwhelming majority of the models assume that the fibres are linearly elastic. While this is a good approximation for many fibre types, it is not generally true. It would cause major errors for oriented polymer fibres, which often show highly non-linear behaviour. Carbon fibres are also known to be non-linear elastic, with its stiffness increasing by about $20 \%$ for every $1 \%$ of applied strain $[101,102]$. This is ignored in nearly all models, although many models are intrinsically capable of incorporating it. In Swolfs et al. [22] for example, the predicted average failure strain for a carbon fibre composite was $2.17 \%$, whereas it would have been $2.42 \%$ if the non-linear elasticity was ignored. It should be noted though that this non-linearity does not affect the predicted strength.

\subsection{Conclusion}

Even if all the input data are accurate, then every model still requires assumptions that may affect the predictions. The influence of fibre packings was found to be negligible, but the 
choice of load sharing rule is an important one. There has been a strong trend to use local load sharing instead of the simplified rules.

The incorporation of dynamic stress concentrations could be a vital step forward. With both experimental and modelling evidence indicating its importance, it is surprising that this has not received more attention. A partial explanation is that modelling dynamic stress redistributions is significantly more challenging than static stress redistributions. It would also require significant adaptations to the strength models and for some of them the adaptations may even be impossible.

The stress redistribution around multiple fibre breaks is receiving more and more attention. Capturing this correctly is crucial for reliable strength models, and good approaches have therefore been developed by several groups. Accurate validations of the superposition rules would be required for cluster sizes close to the critical cluster size.

\section{Concluding remarks}

\subsection{Prediction accuracy}

As explained in "2.1.3. Experimental and statistical issues", several hundreds of tests are required to achieve strength predictions within $10 \%$ of the actual value. Most studies test 25100 fibres, so the expected error is likely to be larger. This is purely based on statistical arguments. Taking into account experimental errors as well, the prediction accuracy cannot be expected to be lower than $20 \%$. This is reasonable, especially in comparison with the prediction accuracy for multidirectional composites under complex loading [2-4].

Nevertheless, many authors have consistently found much better correlations than this $20 \%$ $[23,34,39,118,161,217,218]$. As in many other fields, it is common to continue to improve models and/or input data until they yield a reasonable agreement with experimental results. This human behaviour has been extensively described in the psychology literature, and is termed the "confirmation bias" [219]. It is a natural tendency to look for supporting information and disregard information that contradicts existing data. This is why blind benchmarking studies, such as the World Wide Failure Exercise, are so important in improving model predictions. This type of studies are the best and perhaps the only way to avoid the confirmation bias.

Luckily, the state-of-the-art strength models capture the majority of the mechanisms. These models are therefore powerful tools for carrying out parametric studies and finding optimal combinations. This review has identified areas where the models and their input data can be improved to increase their prediction accuracy. In our opinion, the key areas are:

- More accurate Weibull distributions for fibre strength.

- Reliable measurements of microscale resin properties.

- Incorporation of dynamic stress concentrations.

Improvements in these three areas will be vital for the models to be successful in blind predictions. This type of predictions are much more challenging than predictions where the outcome is known on beforehand.

\subsection{Future trends}

Strength models for UD composites have significantly advanced in the past few decades. They have now reached a point where they can start being useful in real-life applications. Some models have already been built with this specific goal in mind [19,131,220-223], and 
have been applied to composite pressure vessels [136]. This trend is expected to gain significant importance.

Given the increased importance of modelling predictions in composite design, there is also a tendency to incorporate strength models for UD composites into other models. This was for example done by Pimenta and Pinho [224], who used their strength model for UD composites in a model for translaminar fracture toughness. Another example is found in Scott et al. [18], where the effect of transverse ply cracks was taken into account in their strength model for UD composites. This yielded more reliable predictions for a 0/90 laminate.

In the past decades, testing techniques have greatly advanced, and this will also strongly influence the development of strength models for UD composites. Computed tomography can yield new insights into the damage mechanisms [17,21], and provide a useful tool for detailed model validations [18,22]. Further improvements in synchrotron radiation computed tomography will eventually lead to a deeper understanding of the physical mechanisms occurring in UD composites. Specific areas for improvement are:

- Scans at low load levels to measure the Weibull distribution (see "2.1.4. Other testing techniques").

- Shorter hold at loads or continuous data acquisition to minimise time-dependent artifacts (see "2.2.3. Time-dependent aspects").

- Improved algorithms for processing the data, detecting fibre breaks and classifying clusters.

In the nineties, the first FE models for stress redistribution started appearing. Nowadays, the computational power has increased to a point that it becomes possible to build an entire strength model using the FE method $[165,166,216]$. This approach has several advantages, such as the possibility to include fibre misalignment. The drawback at this moment is that it is limited to about 100 fibres, depending on the mesh size.

\section{Acknowledgements}

The authors acknowledge the participants of the benchmarking exercise for strength models of unidirectional composites. The discussions with them were useful to further develop some of the ideas expressed in this review. The authors acknowledge the support of the emeritus Toray chair in Composite Materials for IV. The authors are also grateful for the IWT and FWO Flanders fellowships of YS. YS is currently working at Imperial College London for a 1-year Marie Skłodowska-Curie Fellowship, but remains linked to KU Leuven.

\section{References}

[1] Olave M, Vanaerschot A, Lomov SV, Vandepitte D. Internal geometry variability of two woven composites and related variability of the stiffness. Polymer Composites. 2012;33(8):1335-1350.

[2] Hinton MJ, Kaddour AS, Soden PD. Evaluation of failure prediction in composite laminates: background to 'part C' of the exercise. Composites Science and Technology. 2004;64(3-4):321-327.

[3] Kaddour AS, Hinton MJ. Maturity of 3D failure criteria for fibre-reinforced composites: Comparison between theories and experiments: Part B of WWFE-II. Journal of Composite Materials. 2013;47(6-7):925-966. 
[4] Hinton MJ, Soden PD. Predicting failure in composite laminates: the background to the exercise. Composites Science and Technology. 1998;58(7):1001-1010.

[5] Weibull W. A statistical distribution function of wide applicability. Journal of Applied Mechanics - Transactions of the ASME. 1951;18(3):293-297.

[6] Hedgepeth JM, Van Dyke P. Local stress concentrations in imperfect filamentary composite materials. Journal of Composite Materials. 1967;1(3):294-309.

[7] Hedgepeth JM. Stress concentrations in filamentary structures. NASA TN. 1961;D-882( ):1-36.

[8] Xia Z, Curtin WA, Okabe T. Green's function vs. shear-lag models of damage and failure in fiber composites. Composites Science and Technology. 2002;62(10-11):1279-1288.

[9] Xia Z, Okabe T, Curtin WA. Shear-lag versus finite element models for stress transfer in fiber-reinforced composites. Composites Science and Technology. 2002;62(9):1141-1149.

[10] Fukuda H. Stress concentration factors in unidirectional composites with random fiber spacing. Composites Science and Technology. 1985;22(2):153-163.

[11] Swolfs Y, Gorbatikh L, Romanov V, Orlova S, Lomov SV, Verpoest I. Stress concentrations in an impregnated fibre bundle with random fibre packing. Composites Science and Technology. 2013;74:113-120.

[12] Nedele MR, Wisnom MR. Three-dimensional finite element analysis of the stress concentration at a single fibre break. Composites Science and Technology. 1994;51(4):517524.

[13] Behzadi S, Jones FR. The effect of temperature on stress transfer between a broken fibre and the adjacent fibres in unidirectional fibre composites. Composites Science and Technology. 2008;68(13):2690-2696.

[14] Phoenix SL, Smith RL. A comparison of probabilistic techniques for the strength of fibrous materials under local load-sharing among fibers. International Journal of Solids and Structures. 1983;19(6):479-496.

[15] Smith RL, Phoenix SL, Greenfield MR, Henstenburg RB, Pitt RE. Lower-tail approximations for the probability of failure of three-dimensional fibrous composites with hexagonal geometry Proceedings of the Royal Society of London Series A: Mathematical Physical and Engineering Sciences. 1983;388(1795):353-391.

[16] Swolfs Y, Gorbatikh L, Verpoest I. Stress concentrations in hybrid unidirectional fibrereinforced composites with random fibre packings. Composites Science and Technology. 2013;85:10-16.

[17] Scott AE, Mavrogordato M, Wright P, Sinclair I, Spearing SM. In situ fibre fracture measurement in carbon-epoxy laminates using high resolution computed tomography. Composites Science and Technology. 2011;71:1471-1477.

[18] Scott AE, Sinclair I, Spearing SM, Thionnet A, Bunsell AR. Damage accumulation in a carbon/epoxy composite: Comparison between a multiscale model and computed tomography experimental results. Composites Part A: Applied Science and Manufacturing. 2012;43(9):1514-1522.

[19] Thionnet A, Chou HY, Bunsell A. Fibre break processes in unidirectional composites. Composites Part A: Applied Science and Manufacturing. 2014;65:148-160.

[20] Swolfs Y, Verpoest I, Gorbatikh L. Issues in strength models for unidirectional fibrereinforced composites related to Weibull distributions, fibre packings and boundary effects. Composites Science and Technology. 2015;114:42-49.

[21] Scott AE, Sinclair I, Spearing SM, Mavrogordato MN, Hepples W. Influence of voids on damage mechanisms in carbon/epoxy composites determined via high resolution computed tomography. Composites Science and Technology. 2014;90(0):147-153.

[22] Swolfs Y, Morton H, Scott AE, Gorbatikh L, Reed PAS, Sinclair I, et al. Synchrotron radiation computed tomography for experimental validation of a tensile strength model for 
unidirectional fibre-reinforced composites. Composites Part A: Applied Science and Manufacturing. 2015;77:106-113.

[23] Swolfs Y, McMeeking RM, Verpoest I, Gorbatikh L. Matrix cracks around fibre breaks and their effect on stress redistribution and failure development in unidirectional composites. Composites Science and Technology. 2015;108:16-22.

[24] Rosso A, Moser B, Mortensen A. Tensile strength of axially loaded unidirectional Nextel 610 (TM) reinforced aluminium: A case study in local load sharing between randomly distributed fibres. Composites Part A: Applied Science and Manufacturing. 2012;43(1):129137.

[25] Grail G, Pimenta S, Pinho ST, Robinson P. Exploring the potential of interleaving to delay catastrophic failure in unidirectional composites under tensile loading. Composites Science and Technology. 2015;106(0):100-109.

[26] Timoshenko SP. History of the strength of materials. New York: McGraw-Hill; 1953.

[27] Griffith AA. The Phenomena of Rupture and Flow in Solids1921.

[28] Epstein B. Statistical Aspects of Fracture Problems. Journal of Applied Physics. 1948;19(2):140-147.

[29] Todinov MT. Is Weibull distribution the correct model for predicting probability of failure initiated by non-interacting flaws? International Journal of Solids and Structures. 2009;46(3-4):887-901.

[30] Thomason JL. On the application of Weibull analysis to experimentally determined single fibre strength distributions. Composites Science and Technology. 2013;77:74-80.

[31] Asloun EM, Donnet JB, Guilpain G, Nardin M, Schultz J. On the estimation of the tensile strength of carbon fibres at short lengths. Journal of Materials Science. 1989;24(10):3504-3510.

[32] Behzadi S, Curtis PT, Jones FR. Improving the prediction of tensile failure in unidirectional fibre composites by introducing matrix shear yielding. Composites Science and Technology. 2009;69(14):2421-2427.

[33] Foreman JP, Behzadi S, Porter D, Jones FR. Multi-scale modelling of the effect of a viscoelastic matrix on the strength of a carbon fibre composite. Philosophical Magazine. 2010;90(31-32):4227-4244.

[34] Pimenta S, Pinho ST. Hierarchical scaling law for the strength of composite fibre bundles. Journal of the Mechanics and Physics of Solids. 2013;61(6):1337-1356.

[35] Curtis PT. A computer model of the tensile failure process in unidirectional fibre composites. Composites Science and Technology. 1986;27(1):63-86.

[36] Swolfs Y, McMeeking RM, Gorbatikh L, Verpoest I. The effect of fibre dispersion on initial failure strain and cluster development in unidirectional carbon/glass hybrid composites. Composites Part A: Applied Science and Manufacturing. 2015;69:279-287.

[37] Okabe T, Sekine H, Ishii K, Nishikawa M, Takeda N. Numerical method for failure simulation of unidirectional fiber-reinforced composites with spring element model. Composites Science and Technology. 2005;65(6):921-933.

[38] Okabe T, Takeda N, Kamoshida Y, Shimizu M, Curtin WA. A 3D shear-lag model considering micro-damage and statistical strength prediction of unidirectional fiber-reinforced composites. Composites Science and Technology. 2001;61(12):1773-1787.

[39] Okabe T, Nishikawa M, Takeda N, Sekine H. Effect of matrix hardening on the tensile strength of alumina fiber-reinforced aluminum matrix composites. Acta Materialia. 2006;54(9):2557-2566.

[40] Gulino R, Phoenix SL. Weibull strength statistics for graphite fibres measured from the break progression in a model graphite/glass/epoxy composite. Journal of Materials Science. 1991;26(11):3107-3118. 
[41] Watson AS, Smith RL. An examination of statistical theories for fibrous materials in the light of experimental data. Journal of Materials Science. 1985;20(9):3260-3270.

[42] Beyerlein IJ, Phoenix SL. Statistics for the strength and size effects of microcomposites with four carbon fibers in epoxy resin. Composites Science and Technology. 1996;56(1):7592.

[43] Metcalfe AG, Smitz GK. Effect of length on the strength of glass fibers. Proceedings of the American Society for Testing and Materials. 1964;64:1075-1093.

[44] Phoenix SL, Schwartz P, Robinson HH. Statistics for the strength and lifetime in creeprupture of model carbon/epoxy composites. Composites Science and Technology. 1988;32(2):81-120.

[45] Yu W, Yao J. Tensile strength and its variation of PAN-based carbon fibers. I. Statistical distribution and volume dependence. Journal of Applied Polymer Science. 2006;101(5):31753182.

[46] Chi ZF, Chou TW, Shen GY. Determination of single fibre strength distribution from fibre bundle testing. Journal of Materials Science. 1984;19(10):3319-3324.

[47] Berger MH, Jeulin D. Statistical analysis of the failure stresses of ceramic fibres: Dependence of the Weibull parameters on the gauge length, diameter variation and fluctuation of defect density. Journal of Materials Science. 2003;38(13):2913-2923.

[48] Tanaka T, Nakayama H, Sakaida A, Horikawa N. Estimation of tensile strength distribution for carbon fiber with diameter variation along fiber. Materials Science Research International. 1999;5(2):90-97.

[49] Curtin WA. Tensile strength of fiber-reinforced composites: III. Beyond the traditional Weibull model for fiber strengths. Journal of Composite Materials. 2000;34(15):1301-1332.

[50] Andersons J, Joffe R, Hojo M, Ochiai S. Glass fibre strength distribution determined by common experimental methods. Composites Science and Technology. 2002;62(1):131-145.

[51] Watanabe J, Tanaka F, Okabe T. The tensile strength distribution of carbon fibers at short gauge length. 38th Conference of the Japan Society for Composite Materials, 2013. p. 171-172.

[52] Goda K, Fukunaga H. The evaluation of the strength distribution of silicon-carbide and alumina fibers by a multimodel Weibull distribution. Journal of Materials Science. 1986;21(12):4475-4480.

[53] Yao J, Yu W, Pan D. Tensile Strength and Its Variation of PAN-Based Carbon Fibers. III. Weak-Link Analysis. Journal of Applied Polymer Science. 2008;110(6):3778-3784.

[54] Jones JB, Smith RE, Barr JB. Flaw analysis in high-strength carbon fibers from pitch. Carbon. 1980;18(1):62-62.

[55] Jones JB, Barr JB, Smith RE. Analysis of flaws in high-strength carbon fibers from mesophase pitch. Journal of Materials Science. 1980;15(10):2455-2465.

[56] Loidl D, Paris O, Rennhofer H, Mueller M, Peterlik H. Skin-core structure and bimodal Weibull distribution of the strength of carbon fibers. Carbon. 2007;45(14):2801-2805.

[57] Peterlik H, Loidl D. Bimodal strength distributions and flaw populations of ceramics and fibres. Engineering Fracture Mechanics. 2001;68(3):253-261.

[58] Zinck P, Pays MF, Rezakhanlou R, Gerard JF. Extrapolation techniques at short gauge lengths based on the weakest link concept for fibres exhibiting multiple failure modes. Philosophical Magazine a-Physics of Condensed Matter Structure Defects and Mechanical Properties. 1999;79(9):2103-2122.

[59] Beetz CP. The analysis of carbon fibre strength distributions exhibiting multiple modes of failure. Fibre Science \& Technology. 1982;16(1):45-59.

[60] Stoner EG, Edie DD, Durham SD. An end-effect model for the single-filament tensile test. Journal of Materials Science. 1994;29(24):6561-6574. 
[61] Hitchon JW, Phillips DC. The dependence of the strength of carbon fibres on length. Fibre Science and Technology. 1979;12(3):217-233.

[62] Harlow DG, Phoenix SL. Probability distributions for the strength of composite materials II: A convergent sequence of tight bounds. International Journal of Fracture. 1981;17(6):601630.

[63] Watanabe J, Tanaka F, Okuda H, Okabe T. Tensile strength distribution of carbon fibers at short gauge lengths. Advanced Composite Materials. 2014;23(5-6):535-550.

[64] Okabe T, Takeda N. Size effect on tensile strength of unidirectional CFRP composites experiment and simulation. Composites Science and Technology. 2002;62(15):2053-2064.

[65] Padgett WJ, Durham SD, Mason AM. Weibull analysis of the strength of carbon fibers using linear and power law models for the length effect. Journal of Composite Materials. 1995;29(14):1873-1884.

[66] Gurvich MR, DiBenedetto AT, Ranade SV. A new statistical distribution for characterizing the random strength of brittle materials. Journal of Materials Science. 1997;32(10):2559-2564.

[67] Phani KK. Strength distribution and gauge length extrapolations in glass fibre. Journal of Materials Science. 1988;23(4):1189-1194.

[68] Ibnabdeljalil M, Phoenix SL. Scalings in the statistical failure of brittle matrix composites with discontinuous fibers-I. Analysis and Monte Carlo simulations. Acta Metallurgica et Materialia. 1995;43(8):2975-2983.

[69] Masson JJ, Bourgain E. Some guidelines for a consistent use of the Weibull statistics with ceramic fibres. International Journal of Fracture. 1992;55(4):303-319.

[70] Vanderzwaag S. The concept of filament strength and the Weibull modulus. Journal of Testing and Evaluation. 1989;17(5):292-298.

[71] Yao J, Yu W. Tensile strength and its variation for PAN-based carbon fibers. II. Calibration of the variation from testing. Journal of Applied Polymer Science. 2007;104(4):2625-2632.

[72] Yang L, Thomason JL. Effect of silane coupling agent on mechanical performance of glass fibre. Journal of Materials Science. 2013;48(5):1947-1954.

[73] Bader MG, Priest AM. Statistical aspects of fibre and bundle strength. In: Hayashi T, Kawata K, Umekawa S, editors. Fourth International Conference on Composite Materials, vol. 2 Tokyo, Japan1982. p. 1129-1136.

[74] Phoenix SL, Sexsmith RG. Clamp effects in fiber testing. Journal of Composite Materials. 1972;6(JUL):322-337.

[75] Kelly A, Tyson WR. Tensile properties of fibre-reinforced metals - copper/tungsten and copper/molybdenum. Journal of the Mechanics and Physics of Solids. 1965;13(6):329-350.

[76] Zinck P, Mader E, Gerard JF. Role of silane coupling agent and polymeric film former for tailoring glass fiber sizings from tensile strength measurements. Journal of Materials Science. 2001;36(21):5245-5252.

[77] Naito K, Yang JM, Tanaka Y, Kagawa Y. The effect of gauge length on tensile strength and Weibull modulus of polyacrylonitrile (PAN)- and pitch-based carbon fibers. Journal of Materials Science. 2012;47(2):632-642.

[78] Pimenta S, Pinho ST. The influence of micromechanical properties and reinforcement architecture on the mechanical response of recycled composites. Composites Part A: Applied Science and Manufacturing. 2014;56:213-225.

[79] Li CT, Langley NR. Improvement in fiber testing of high-modulus single-filament materials. Journal of the American Ceramic Society. 1985;68(8):C202-C204.

[80] Bermejo R, Supancic P, Danzer R. Influence of measurement uncertainties on the determination of the Weibull distribution. Journal of the European Ceramic Society. 2012;32(2):251-255. 
[81] Naito K, Tanaka Y, Yang J-M, Kagawa Y. Tensile properties of ultrahigh strength PANbased, ultrahigh modulus pitch-based and high ductility pitch-based carbon fibers. Carbon. 2008;46(2):189-195.

[82] Naito K, Yang J-M, Kagawa Y. Tensile properties of high strength polyacrylonitrile (PAN)-based and high modulus pitch-based hybrid carbon fibers-reinforced epoxy matrix composite. Journal of Materials Science. 2012;47(6):2743-2751.

[83] Parthasarathy TA. Extraction of Weibull parameters of fiber strength from means and standard deviations of failure loads and fiber diameters. Journal of the American Ceramic Society. 2001;84(3):588-592.

[84] Blassiau S. Modélisation des phénomènes microstructuraux au sein d'un composite unidirectionnel carbone/epoxy et prédiction de durée de vie : contrôle et qualification de réservoirs bobinés. École Nationale Supérieure des Mines de Paris, 2005.

[85] Lara-Curzio E, Russ CM. Why it is necessary to determine each fiber diameter when estimating the parameters of the distribution of fiber strengths. Ceramic Engineering and Science Proceedings. 1999;20(3):471-480.

[86] Lara-Curzio E, Russ CM. On the relationship between the parameters of the distributions of fiber diameters, breaking loads, and fiber strengths. Journal of Materials Science Letters. 1999;18(24):2041-2044.

[87] Shioya M, Takaku A. Estimation of fibre and interfacial shear strength by using a singlefibre composite. Composites Science and Technology. 1995;55(1):33-39.

[88] Verpoest I, Desaeger M, Ivens J, Wevers M. Interfaces in polymer matrix composites from micromechanical tests to macromechanical properties. Makromolekulare ChemieMacromolecular Symposia. 1993;75:85-98.

[89] Wu W, Desaeger M, Verpoest I, Varna J. An improved analysis of the stresses in a single-fibre fragmentation test .1. Two-phase model. Composites Science and Technology. 1997;57(7):809-819.

[90] Fraser AA, Ancker FH, Dibenedetto AT. A Computer Modelled Single Filament Technique for Measuring Coupling and Sizing Agent Effects in Fibre Reinforced Composites. 30th Annual technical conference on reinforced plastics, vol. 22-A: The Society of Plastics Industry; 1975.

[91] Lacroix T, Keunings R, Desaeger M, Verpoest I. A new data reduction scheme for the fragmentation testing of polymer composites. Journal of Materials Science. 1995;30(3):683692.

[92] Lachman N, Carey BJ, Hashim DP, Ajayan PM, Wagner HD. Application of continuously-monitored single fiber fragmentation tests to carbon nanotube/carbon microfiber hybrid composites. Composites Science and Technology. 2012;72(14):1711-1717.

[93] Zhao FM, Okabe T, Takeda N. The estimation of statistical fiber strength by fragmentation tests of single-fiber composites. Composites Science and Technology. 2000;60(10):1965-1974.

[94] Hui CY, Phoenix SL, Shia D. The single-filament-composite test: A new statistical theory for estimating the interfacial shear strength and Weibull parameters for fiber strength. Composites Science and Technology. 1997;57(12):1707-1725.

[95] Okabe T, Takeda N. Elastoplastic shear-lag analysis of single-fiber composites and strength prediction of unidirectional multi-fiber composites. Composites Part A: Applied Science and Manufacturing. 2002;33(10):1327-1335.

[96] Detassis M, Pegoretti A, Migliaresi C, Wagner HD. Experimental evaluation of residual stresses in single fibre composites by means of the fragmentation test. Journal of Materials Science. 1996;31(9):2385-2392. 
[97] Okuda H, Young RJ, Tanaka F, Watanabe J, Okabe T. The tensile strength of PAN-based carbon fibres and nanostructure development. 20th International Conference on Composite Materials, Copenhagen, Denmark2015.

[98] Sinclair D. A Bending Method for Measurement of the Tensile Strength and Young's Modulus of Glass Fibers. Journal of Applied Physics. 1950;21(5):380-386.

[99] Fukuda H, Yakushiji M, Wada A. A loop test to measure the strength of monofilaments used for advanced composites. Advanced Composite Materials. 1999;8(3):281-291.

[100] Williams WS, Steffens DA, Bacon R. Bending behavior and tensile strength of carbon fibers. Journal of Applied Physics. 1970;41(12):4893-4901.

[101] Nukushina Y, Matsui J, Itoh M. On the Tensile Load to Elongation Response of Carbon Fibre Single Filament Samples. J Jap Soc Compos Mater. 1989;15(5):210-221.

[102] Toyama N, Takatsubo J. An investigation of non-linear elastic behavior of CFRP laminates and strain measurement using Lamb waves. Composites Science and Technology. 2004;64(16):2509-2516.

[103] Jones WR, Johnson JW. Intrinsic strength and non-hookean behaviour of carbon fibres. Carbon. 1971;9(5):645-655.

[104] van den Heuvel PWJ, Goutianos S, Young RJ, Peijs T. Failure phenomena in fibrereinforced composites. Part 6: a finite element study of stress concentrations in unidirectional carbon fibre-reinforced epoxy composites. Composites Science and Technology. 2004;64(5):645-656.

[105] van den Heuvel PWJ, Wubbolts MK, Young RJ, Peijs T. Failure phenomena in twodimensional multi-fibre model composites: 5. A finite element study. Composites Part AApplied Science and Manufacturing. 1998;29(9-10):1121-1135.

[106] Xia Z, Curtin WA, Peters PWM. Multiscale modeling of failure in metal matrix composites. Acta Materialia. 2001;49(2):273-287.

[107] Cox HL. The elasticity and strength of paper and other fibrous materials. British Journal of Applied Physics. 1952;3(3):72-79.

[108] Du ZZ, McMeeking RM. Creep models for metal matrix composites with long brittle fibers. Journal of the Mechanics and Physics of Solids. 1995;43(5):701-726.

[109] Ohno N, Ando T, Miyake T, Biwa S. A variational method for unidirectional fiberreinforced composites with matrix creep. International Journal of Solids and Structures. 2002;39(1):159-174.

[110] Landis CM, McMeeking RM. A shear-lag model for a broken fiber embedded in a composite with a ductile matrix. Composites Science and Technology. 1999;59(3):447-457.

[111] Mahesh S, Hanan JC, Ustundag E, Beyerlein IJ. Shear-lag model for a single fiber metal matrix composite with an elasto-plastic matrix and a slipping interface. International Journal of Solids and Structures. 2004;41(15):4197-4218.

[112] de Morais AB. Stress distribution along broken fibres in polymer-matrix composites. Composites Science and Technology. 2001;61(11):1571-1580.

[113] Nedele MR, Wisnom MR. Stress-Concentration Factors around a Broken Fiber in a Unidirectional Carbon-Fiber-Reinforced Epoxy. Composites. 1994;25(7):549-557.

[114] Fiedler B, Klisch A, Schulte K. Stress concentrations in multiple fibre model composites. Composites Part A: Applied Science and Manufacturing. 1998;29(9-10):10131019.

[115] Accorsi ML, Pegoretti A, Dibenedetto AT. Dynamic analysis of fibre breakage in single- and multiple-fibre composites. Journal of Materials Science. 1996;31(16):4181-4187.

[116] Lane R, Hayes SA, Jones FR. Fibre/matrix stress transfer through a discrete interphase:

2. High volume fraction systems. Composites Science and Technology. 2001;61(4):565-578.

[117] Xia ZH, Curtin WA. Multiscale modeling of damage and failure in aluminum-matrix composites. Composites Science and Technology. 2001;61(15):2247-2257. 
[118] de Morais AB. Prediction of the longitudinal tensile strength of polymer matrix composites. Composites Science and Technology. 2006;66(15):2990-2996.

[119] Brosh T, Ganor Y, Belov I, Pilo R. Analysis of strength properties of light-cured resin composites. Dental Materials. 1999;15(3):174-179.

[120] Fiedler B, Hojo M, Ochiai S, Schulte K, Ando M. Failure behavior of an epoxy matrix under different kinds of static loading. Composites Science and Technology. 2001;61(11):1615-1624.

[121] Giannotti MI, Galante MJ, Oyanguren PA, Vallo CI. Role of intrinsic flaws upon flexural behaviour of a thermoplastic modified epoxy resin. Polymer Testing. 2003;22(4):429437.

[122] Ben Salem N, Bresson G, Jumel J, Shanahan MER, Bellut S, Lavelle F. Weibull analysis of stiffness and strength in bulk epoxy adhesives reinforced with particles. Journal of Adhesion Science and Technology. 2013;27(21):2278-2295.

[123] Callens MG, Gorbatikh L, Verpoest I. Ductile steel fibre composites with brittle and ductile matrices. Composites Part A: Applied Science and Manufacturing. 2014;61:235-244.

[124] Odom EM, Adams DF. Specimen size effect during tensile testing of an unreinforced polymer. Journal of Materials Science. 1992;27(7):1767-1771.

[125] Towse A, Potter K, Wisnom MR, Adams RD. Specimen size effects in the tensile failure strain of an epoxy adhesive. Journal of Materials Science. 1998;33(17):4307-4314.

[126] Hobbiebrunken T, Fiedler B, Hojo M, Tanaka M. Experimental determination of the true epoxy resin strength using micro-scaled specimens. Composites Part A: Applied Science and Manufacturing. 2007;38(3):814-818.

[127] Misumi J, Ganesh R, Sockalingam S, Gillespie Jr JW. The role of matrix resin microscale properties on the axial tensile strength of CFRP. 20th International Conference on Composite Materials, Copenhagen, Denmark2015.

[128] Iyengar N, Curtin WA. Time-dependent failure in fiber-reinforced composites by matrix and interface shear creep. Acta Materialia. 1997;45(8):3419-3429.

[129] Lagoudas DC, Chung-Yuen H, Phoenix SL. Time evolution of overstress profiles near broken fibers in a composite with a viscoelastic matrix. International Journal of Solids and Structures. 1989;25(1):45-66.

[130] Mason DD, Chung-Yuen H, Phoenix SL. Stress profiles around a fiber break in a composite with a nonlinear, power law creeping matrix. International Journal of Solids and Structures. 1992;29(23):2829-2854.

[131] Blassiau S, Thionnet A, Bunsell AR. Micromechanisms of load transfer in a unidirectional carbon fibre-reinforced epoxy composite due to fibre failures. Part 2: Influence of viscoelastic and plastic matrices on the mechanisms of load transfer. Composite Structures. 2006;74(3):319-331.

[132] Lifshitz JM, Rotem A. Time-dependent longitudinal strength of unidirectional fibrous composites. Fibre Science and Technology. 1970;3(1):1-20.

[133] Fabeny B, Curtin WA. Damage-enhanced creep and rupture in fiber-reinforced composites. Acta Materialia. 1996;44(9):3439-3451.

[134] Beyerlein IJ, Phoenix SL, Raj R. Time evolution of stress redistribution around multiple fiber breaks in a composite with viscous and viscoelastic matrices. International Journal of Solids and Structures. 1998;35(24):3177-3211.

[135] Otani H, Phoenix SL, Petrina P. Matrix effects on lifetime statistics for carbon fiber/epoxy microcomposites in creep-rupture. Journal of Materials Science. 1991;26(7):1955-1970.

[136] Chou HY, Bunsell AR, Mair G, Thionnet A. Effect of the loading rate on ultimate strength of composites. Application: Pressure vessel slow burst test. Composite Structures. 2013;104(0):144-153. 
[137] Foreman JP, Behzadi S, Tsampas SA, Porter D, Curtis PT, Jones FR. Rate dependent multiscale modelling of fibre reinforced composites. Plast Rubber Compos. 2009;38(2-4):6771.

[138] Ochola RO, Marcus K, Nurick GN, Franz T. Mechanical behaviour of glass and carbon fibre reinforced composites at varying strain rates. Composite Structures. 2004;63(3-4):455467.

[139] McLean M. Creep deformation of metal-matrix composites. Composites Science and Technology. 1985;23(1):37-52.

[140] Detassis M, Frydman E, Vrieling D, Zhou XF, Wagner HD, Nairn JA. Interface toughness in fibre composites by the fragmentation test. Composites Part A: Applied Science and Manufacturing. 1996;27(9):769-773.

[141] Lacroix T, Tilmans B, Keunings R, Desaeger M, Verpoest I. Modeling of critical fiber length and interfacial debonding in the fragmentation testing of polymer composites. Composites Science and Technology. 1992;43(4):379-387.

[142] Paipetis A, Galiotis C, Liu YC, Nairn JA. Stress transfer from the matrix to the fibre in a fragmentation test: Raman experiments and analytical modeling. Journal of Composite Materials. 1999;33(4):377-399.

[143] Zhao FM, Takeda N. Effect of interfacial adhesion and statistical fiber strength on tensile strength of unidirectional glass fiber/epoxy composites. Part I: experiment results. Composites Part A-Applied Science and Manufacturing. 2000;31(11):1203-1214.

[144] Madhukar MS, Drzal LT. Fiber-Matrix Adhesion and Its Effect on Composite Mechanical Properties: II. Longitudinal $\left(0^{\circ}\right)$ and Transverse $\left(90^{\circ}\right)$ Tensile and Flexure Behavior of Graphite/Epoxy Composites. Journal of Composite Materials. 1991;25(8):958991.

[145] Shih GC, Ebert LJ. Theoretical Modelling of the Effect of the Interfacial Shear Strength on the Longitudinal Tensile Strength of Unidirectional Composites. Journal of Composite Materials. 1987;21(3):207-224.

[146] Zhandarov S, Mäder E. Characterization of fiber/matrix interface strength: applicability of different tests, approaches and parameters. Composites Science and Technology. 2005;65(1):149-160.

[147] Herrera-Franco PJ, Drzal LT. Comparison of methods for the measurement of fibre/matrix adhesion in composites. Composites. 1992;23(1):2-27.

[148] Pitkethly MJ, Favre JP, Gaur U, Jakubowski J, Mudrich SF, Caldwell DL, et al. A round-robin programme on interfacial test methods. Composites Science and Technology. 1993;48(1-4):205-214.

[149] Netravali AN, Henstenburg RB, Phoenix SL, Schwartz P. Interfacial shear strength studies using the single-filament-composite test. 1. Experiments on graphite fibers in epoxy. Polymer Composites. 1989;10(4):226-241.

[150] Gao X, Gillespie JW, Jensen RE, Li W, Haque BZ, McKnight SH. Effect of fiber surface texture on the mechanical properties of glass fiber reinforced epoxy composite. Composites Part a-Applied Science and Manufacturing. 2015;74:10-17.

[151] Lei ZK, Qiu W, Kang YL, Gang L, Yun H. Stress transfer of single fiber/microdroplet tensile test studied by micro-Raman spectroscopy. Composites Part a-Applied Science and Manufacturing. 2008;39(1):113-118.

[152] Penn LS, Bowler ER. A new approach to surface energy characterization for adhesive performance prediction. Surface and Interface Analysis. 1981;3(4):161-164.

[153] Chua PS, Piggott MR. The glass fibre-polymer interface: I-theoretical consideration for single fibre pull-out tests. Composites Science and Technology. 1985;22(1):33-42.

[154] Gonzalez C, Llorca J. Micromechanical modelling of deformation and failure in Ti-6Al4V/SiC composites. Acta Materialia. 2001;49(17):3505-3519. 
[155] Zhou XF, Wagner HD, Nutt SR. Interfacial properties of polymer composites measured by push-out and fragmentation tests. Composites Part A: Applied Science and Manufacturing. 2001;32(11):1543-1551.

[156] Zhao FM, Takeda N. Effect of interfacial adhesion and statistical fiber strength on tensile strength of unidirectional glass fiber/epoxy composites. Part. II: comparison with prediction. Composites Part A-Applied Science and Manufacturing. 2000;31(11):1215-1224.

[157] Zweben C. Tensile strength of hybrid composites. Journal of Materials Science. 1977;12(7):1325-1337.

[158] Zweben C. Tensile failure of fiber composites. AIAA Journal. 1968;6(12):2325-2331.

[159] Scop PM, Argon AS. Statistical theory of strength of laminated composites II. Journal of Composite Materials. 1969;3(JAN):30-47.

[160] Goree JG, Gross RS. Analysis of a unidirectional composite containing broken fibers and matrix damage. Engineering Fracture Mechanics. 1980;13(3):563-578.

[161] Okabe T, Ishii K, Nishikawa M, Takeda N. Prediction of Tensile Strength of Unidirectional CFRP Composites. Advanced Composite Materials. 2010;19(3):229-241.

[162] Mahesh S, Phoenix SL, Beyerlein IJ. Strength distributions and size effects for 2D and $3 \mathrm{D}$ composites with Weibull fibers in an elastic matrix. International Journal of Fracture. 2002;115(1):41-85.

[163] Landis CM, McMeeking RM. Stress concentrations in composites with interface sliding, matrix stiffness and uneven fiber spacing using shear lag theory. International Journal of Solids and Structures. 1999;36(28):4333-4361.

[164] Landis CM, McGlockton MA, McMeeking RM. An improved shear lag model for broken fibers in composite materials. Journal of Composite Materials. 1999;33(7):667-680.

[165] Mishnaevsky Jr L, Brøndsted P. Micromechanisms of damage in unidirectional fiber reinforced composites: 3D computational analysis. Composites Science and Technology. 2009;69(7-8):1036-1044.

[166] Mishnaevsky Jr L, Dai G. Hybrid carbon/glass fiber composites: Micromechanical analysis of structure-damage resistance relationships. Computational Materials Science. 2014;81:630-640.

[167] Curtin WA, Takeda N. Tensile strength of fiber-reinforced composites: I. Model and effects of local fiber geometry. Journal of Composite Materials. 1998;32(22):2042-2059.

[168] Romanov V, Lomov SV, Swolfs Y, Orlova S, Gorbatikh L, Verpoest I. Statistical analysis of real and simulated fibre arrangements in unidirectional composites. Composites Science and Technology. 2013;87:126-134.

[169] Wongsto A, Li S. Micromechanical FE analysis of UD fibre-reinforced composites with fibres distributed at random over the transverse cross-section. Composites Part A: Applied Science and Manufacturing. 2005;36(9):1246-1266.

[170] Trujillo E, Moesen M, Osorio L, Van Vuure AW, Ivens J, Verpoest I. Bamboo fibres for reinforcement in composite materials: Strength Weibull analysis. Composites Part A: Applied Science and Manufacturing. 2014;61(0):115-125.

[171] Hine PJ, Bonner M, Ward IM, Swolfs Y, Verpoest I, Mierzwa A. Hybrid carbon fibre/nylon 12 single polymer composites. Composites Part A: Applied Science and Manufacturing. 2014;65:19-26.

[172] Swolfs Y, Czél G, Jalalvand M, Wisnom MR, Verpoest I, Gorbatikh L. Model validation of the hybrid effect in carbon/glass hybrid composites. In: González C, López C, Llorca J, editors. 7th International Conference on Composites Testing and Model Identification, Madrid, Spain: IMDEA; 2015.

[173] Harlow DG, Phoenix SL. The chain-of-bundles probability model for the strength of fibrous materials I: Analysis and conjectures. Journal of Composite Materials. 1978;12(APR):195-214. 
[174] Harlow DG, Phoenix SL. The chain-of-bundles probability model for the strength of fibrous materials II: Numerical study of convergence. Journal of Composite Materials. 1978;12(JUL):314-334.

[175] Harlow DG, Phoenix SL. Probability distributions for the strength of composite materials I: two-level bounds. International Journal of Fracture. 1981;17(4):347-372.

[176] Pitt RE, Phoenix SL. Probability distributions for the strength of composite materials.

III: The effect of fiber arrangement. International Journal of Fracture. 1982;20(4):291-311.

[177] Smith RL. Limit theorems and approximations for the reliability of load-sharing systems. Advances in Applied Probability. 1983;15(2):304-330.

[178] Duxbury PM, Leath PL. Exactly solvable models of material breakdown. Physical Review B. 1994;49(18):12676-12687.

[179] Zhou XF, Wagner HD. Stress concentrations caused by fiber failure in two-dimensional composites. Composites Science and Technology. 1999;59(7):1063-1071.

[180] Rosen BW. Tensile failure of fibrous composites. AIAA Journal. 1964;2(11):19851991.

[181] Curtin WA. The "tough" to brittle transition in brittle matrix composites. Journal of the Mechanics and Physics of Solids. 1993;41(2):217-245.

[182] Neumeister JM. A constitutive law for continuous fiber reinforced brittle matrix composites with fiber fragmentation and stress recovery. Journal of the Mechanics and Physics of Solids. 1993;41(8):1383-1404.

[183] Neumeister JM. Bundle pullout-a failure mechanism limiting the tensile strength of continuous fiber reinforced brittle matrix composites - and its implications for strength dependence on volume and type of loading. Journal of the Mechanics and Physics of Solids. 1993;41(8):1405-1424.

[184] Hui CY, Phoenix SL, Ibnabdeljalil M, Smith RL. An exact closed form solution for fragmentation of Weibull fibers in a single filament composite with applications to fiberreinforced ceramics. Journal of the Mechanics and Physics of Solids. 1995;43(10):1551-1585. [185] Rajan VP, Zok FW. Effects of non-uniform strains on tensile fracture of fiberreinforced ceramic composites. Journal of the Mechanics and Physics of Solids. 2012;60(12):2003-2018.

[186] Curtin WA. Theory of mechanical properties of ceramic-matrix composites. Journal of the American Ceramic Society. 1991;74(11):2837-2845.

[187] Curtin WA. Exact theory of fibre fragmentation in a single-filament composite. Journal of Materials Science. 1991;26(19):5239-5253.

[188] Swolfs Y, McMeeking RM, Rajan VP, Zok FW, Verpoest I, Gorbatikh L. Global load sharing model for unidirectional hybrid fibre-reinforced composites. Journal of the Mechanics and Physics of Solids. 2015;84:380-394.

[189] Rajan VP, Curtin WA. Rational design of fiber-reinforced hybrid composites: A global load sharing analysis. Composites Science and Technology. 2015;117:199-207.

[190] Okabe T, Nishikawa M. GLS strength prediction of glass-fiber-reinforced polypropylene. Journal of Materials Science. 2008;44(1):331-334.

[191] He MY, Evans AG, Curtin WA. The ultimate tensile strength of metal and ceramicmatrix composites. Acta Metallurgica et Materialia. 1993;41(3):871-878.

[192] Curtin WA. Stochastic damage evolution and failure in fiber-reinforced composites. Advances in Applied Mechanics, Vol 36. 1998;36:163-253.

[193] Zhou SJ, Curtin WA. Failure of fiber composites: a lattice green function model. Acta Metallurgica Et Materialia. 1995;43(8):3093-3104.

[194] van den Heuvel PWJ, van der Bruggen YJW, Peijs T. The influence of carbon fibre surface treatment on fibre-fibre interactions in multi-fibre microcomposites. Adv Compos Lett. 1994;3(6):197-201. 
[195] Jones KD, Dibenedetto AT. Fiber fracture in hybrid composite systems. Composites Science and Technology. 1994;51(1):53-62.

[196] Ji X, Liu XR, Chou TW. Dynamic stress concentration factors in unidirectional composites. Journal of Composite Materials. 1985;19(3):269-275.

[197] Sakharova EN, Ovchinskii AS. Influence of dynamic effects accompanying rupture of fibers and separation of fibers from the matrix on interaction between failure micromechanisms of composite materials. Mech Compos Mater. 1984;20(3):323-327.

[198] Sakharova EN, Ovchinskii AS. Dynamics of stress redistribution in fiber fracture in composites. Mech Compos Mater. 1980;16(4):417-422.

[199] Sakharova EN, Ovchinskii AS. Dynamics of stress redistribution in a ruptured fiber of a composition material. Mech Compos Mater. 1979;15(1):45-51.

[200] Xing J, Hsiao GC, Chou TW. A dynamic explanation of the hybrid effect. Journal of Composite Materials. 1981;15(SEP):443-461.

[201] Swolfs Y, Gorbatikh L, Verpoest I. Fibre hybridisation in polymer composites: a review. Composites Part A: Applied Science and Manufacturing. 2014;67:181-200.

[202] Goree JG, Gross RS. Stresses in a three-dimensional unidirectional composite containing broken fibers. Engineering Fracture Mechanics. 1980;13(2):395-405.

[203] Ochiai S, Schulte K, Peters PWM. Strain concentration factors for fibers and matrix in unidirectional composites. Composites Science and Technology. 1991;41(3):237-256.

[204] Fukuda H, Chou TW. An advanced shear-lag model applicable to discontinuous fiber composites. Journal of Composite Materials. 1981;15(JAN):79-91.

[205] Beyerlein IJ, Phoenix SL. Stress concentrations around multiple fiber breaks in an elastic matrix with local yielding or debonding using quadratic influence superposition. Journal of the Mechanics and Physics of Solids. 1996;44(12):1997-2039.

[206] Sastry AM, Phoenix SL. Load redistribution near non-aligned fiber breaks in a 2dimensional unidirectional composite using break-influence superposition. Journal of Materials Science Letters. 1993;12(20):1596-1599.

[207] Li H, Jia Y, Mamtimin G, Jiang W, An L. Stress transfer and damage evolution simulations of fiber-reinforced polymer-matrix composites. Materials Science and Engineering A. 2006;425(1-2):178-184.

[208] Kachanov M. Elastic solids with many cracks: a simple method of analysis. International Journal of Solids and Structures. 1987;23(1):23-43.

[209] Kachanov M. A simple technique of stress analysis in elastic solids with many cracks. International Journal of Fracture. 1985;28(1):R11-R19.

[210] Mishnaevskyjr L, Brondsted P. Micromechanical modeling of damage and fracture of unidirectional fiber reinforced composites: A review. Computational Materials Science. 2009;44(4):1351-1359.

[211] Aveston J, Kelly A. Theory of Multiple Fracture of Fibrous Composites. Journal of Materials Science. 1973;8(3):352-362.

[212] Aveston J, Cooper GA, Kelly A. The properties of fibre composites. Conference proceedings of the National Physical Laboratory, Surrey, United Kingdom: IPC Science and Technology Press Ltd.; 1971.

[213] Budiansky B, Hutchinson JW, Evans AG. Matrix fracture in fiber-reinforced ceramics. Journal of the Mechanics and Physics of Solids. 1986;34(2):167-189.

[214] Wisnom MR. The effect of fibre misalignment on the compressive strength of unidirectional carbon fibre/epoxy. Composites. 1990;21(5):403-407.

[215] Yurgartis SW. Measurement of small angle fiber misalignments in continuous fiber composites. Composites Science and Technology. 1987;30(4):279-293.

[216] Dai G, Mishnaevsky Jr L. Fatigue of hybrid glass/carbon composites: 3D computational studies. Composites Science and Technology. 2014;94:71-79. 
[217] Curtin WA, Takeda N. Tensile strength of fiber-reinforced composites: II. Application to polymer matrix composites. Journal of Composite Materials. 1998;32(22):2060-2081.

[218] Noda J, Nakada M, Miyano Y. Temperature dependence of accumulation of fiber breakages under tensile loading for unidirectional CFRP laminates. Journal of Reinforced Plastics and Composites. 2008;27(10):1005-1019.

[219] Jonas E, Schulz-Hardt S, Frey D, Thelen N. Confirmation bias in sequential information search after preliminary decisions: An expansion of dissonance theoretical research on selective exposure to information. Journal of Personality and Social Psychology. 2001;80(4):557-571.

[220] Blassiau S, Bunsell AR, Thionnet A. Damage accumulation processes and life prediction in unidirectional composites. Proceedings of the Royal Society of London Series A: Mathematical Physical and Engineering Sciences. 2007;463(2080):1135-1152.

[221] Blassiau S, Thionnet A, Bunsell AR. Three-dimensional analysis of load transfer micromechanisms in fibre/matrix composites. Composites Science and Technology. 2009;69(1):3337.

[222] Blassiau S, Thionnet A, Bunsell AR. Micromechanisms of load transfer in a unidirectional carbon fibre-reinforced epoxy composite due to fibre failures: Part 3 . Multiscale reconstruction of composite behaviour. Composite Structures. 2008;83(3):312323.

[223] Blassiau S, Thionnet A, Bunsell AR. Micromechanisms of load transfer in a unidirectional carbon fibre-reinforced epoxy composite due to fibre failures. Part 1: Micromechanisms and 3D analysis of load transfer: The elastic case. Composite Structures. 2006;74(3):303-318.

[224] Pimenta S, Pinho ST. An analytical model for the translaminar fracture toughness of fibre composites with stochastic quasi-fractal fracture surfaces. Journal of the Mechanics and Physics of Solids. 2014;66(0):78-102. 